\title{
Self-Assembly under Confinement: Nanocorrals for Understanding Fundamentals of 2D Crystallization
}

\author{
Lander Verstraete, John Greenwood, ${ }^{* \dagger}$ Brandon E. Hirsch, and Steven De Feyter*
}

Department of Chemistry, Division of Molecular Imaging and Photonics, KU Leuven-University of Leuven, Celestijnenlaan 200F, B-3001 Leuven, Belgium

\section{Supporting Information}

ABSTRACT: Nanocorrals with different size, shape, and orientation are created on covalently modified highly oriented pyrolytic graphite surfaces using scanning probe nanolithography, i.e., nanoshaving. Alkylated diacetylene molecules undergo laterally confined supramolecular selfassembly within these corrals. When nanoshaving is performed in situ, at the liquid-solid interface, the orientation of the supramolecular lamellae structure is directionally influenced by the gradual graphite surface exposure. Careful choice of the nanoshaving direction with respect to the substrate symmetry axes promotes alignment

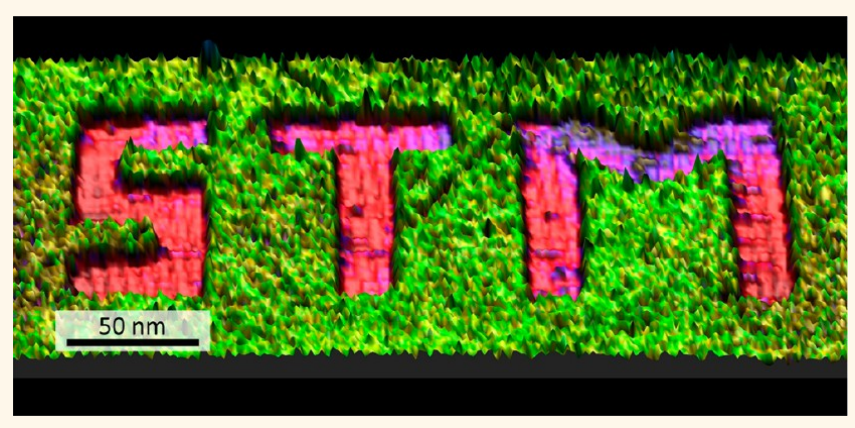
of the supramolecular lamellae within the corral. Selfassembly occurring inside corrals of different size and shape reveals the importance of geometric and kinetic constraints controlled by the nanoshaving process. Finally, seed-mediated crystallization studies demonstrate confinement control over nucleation and growth principles.

KEYWORDS: self-assembly, confinement, nucleation and growth, scanning tunneling microscopy

upramolecular self-assembly on surfaces is an active area of research aimed toward the realization of a range of (functional) two-dimensional (2D) crystals. ${ }^{1-4}$ Control over the network morphology and defect density is a necessity for precise engineering of these materials. Formation of highquality interfaces is important for engineering organic thin-film devices with $\pi$-conjugated molecules, where the relative orientation of molecules is crucial to enable efficient charge transport across electrodes. ${ }^{5,6}$ Great efforts are taken to improve the quality of $2 \mathrm{D}$ crystals, ${ }^{7-9}$ by avoiding molecular defects and limiting domain boundaries. A host of variables are available to achieve this, either intrinsically (molecular design and symmetry) ${ }^{10}$ or extrinsically (temperature, ${ }^{11,12}$ solute concentration, $^{13}$ type of solvent, ${ }^{14,15}$ capillary flow, ${ }^{16}$ and substrate). ${ }^{17}$ Despite the tunability of these parameters, the time scales and complexity of molecular recognition and assembly processes have thus far limited a complete comprehension and control of the $2 \mathrm{D}$ crystal formation. As such, studies targeting the fundamental understanding of kinetic and thermodynamic parameters within these systems are rare. ${ }^{11,18-20}$ An ability to manipulate nucleation and growth processes would afford greater control over network formation and possibly a more detailed molecular level understanding of assembly principles.
One approach to study the elementary aspects of selfassembly consists of compartmentalizing the assembling molecules into laterally confined areas. Beebe et al. demonstrated that by heating highly oriented pyrolytic graphite (HOPG) in the presence of oxygen, thermally oxidized pits can be etched into the surface. ${ }^{21}$ These pits were proven to be useful tools for molecular self-assembly confinement studies; however their preparation methods limit control over the shape, dimensionality, or orientation with respect to the symmetry axes of the underlying substrate lattice. ${ }^{22,23}$ Other studies within confined spaces have been reported by de Oteyza et al., who showed that under ultra-high-vacuum conditions, self-assembly of diindenoperylene on $\mathrm{Cu}$ (111) step edges leads to the formation of a long-range ordered structure with codirectionally oriented molecules. ${ }^{24}$ Alignment is observed along the confined step edges of the Ag (877) vicinal surface for terminal alkyne-functionalized polyphenylene building blocks. ${ }^{25}$ Studies targeting confinement at very small length scales ${ }^{26}$ (a few nanometers) have demonstrated the role of electronic surface states using well-organized adsorbates. ${ }^{27-29}$ Never-

Received: September 3, 2016

Accepted: October 11, 2016

Published: October 17, 2016 
A

HOPG

\section{covalent modification}

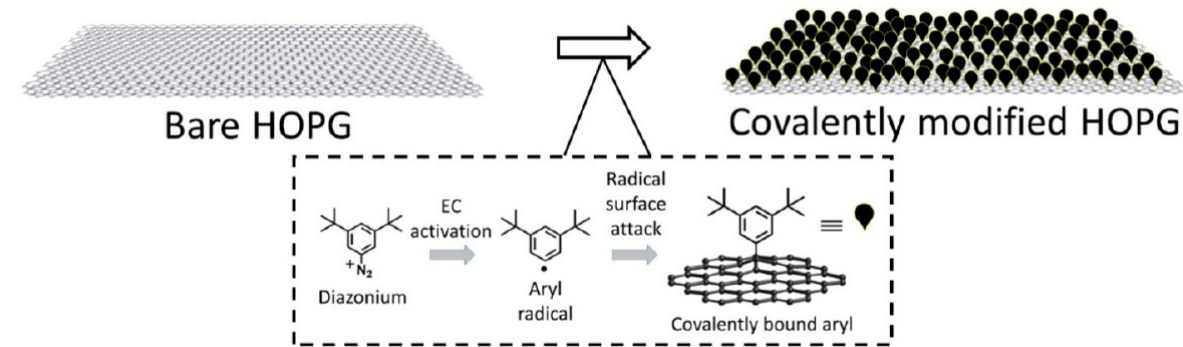

B Nanocorral formation via STM

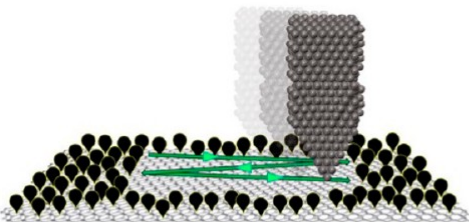

Ex situ

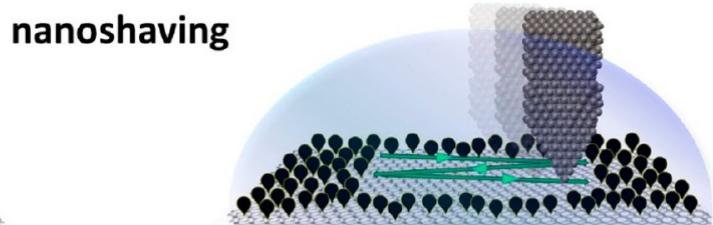

In situ

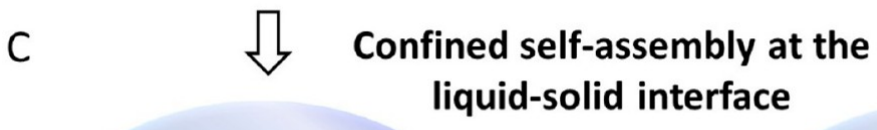

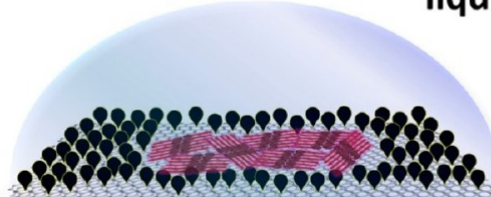

No PCDA alignment

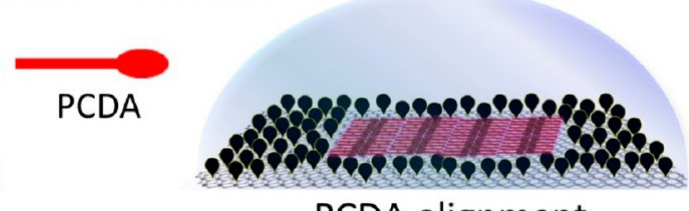

PCDA alignment

Figure 1. Schematic illustration of the covalent modification of the HOPG surface, nanoshaving performed by the STM tip (ex situ and in situ) to generate the nanocorrals, and the subsequent molecular self-assembly studies at the liquid-solid interface using PCDA. The PCDA lamellae alignment is controlled by nanoshaving within in situ created nanocorrals. Corrals formed $e x$ situ do not display the same alignment behavior.

theless, alternative approaches that yield well-defined corrals for studying molecular self-assembly under nanoconfinement may afford greater insight into the fundamentals of recognition and growth processes.

In this work, we create confined spaces (nanocorrals) with geometric size, shape, and orientational control on covalently modified HOPG surfaces. Corral production involves two separate steps. First, HOPG is covalently modified by aryl radicals that are electrochemically (EC) generated from an aryl diazonium precursor species (Figure 1a). ${ }^{30-33}$ In the second step, the tip of a scanning tunneling microscope (STM) is used to precisely remove the covalently bound species from the surface, a process that is referred to as "nanoshaving" (Figure 1b). Subsequent self-assembly investigations (Figure 1c) within these well-defined nanocorrals are carried out at the liquidsolid interface using a saturated solution of 10,12-pentacosadiynoic acid (PCDA) in 1-phenyloctane (1-PO). The PCDA molecule contains typical structural and functional features such as alkyl chains and a carboxylic acid headgroup that are known to foster supramolecular self-assembly at the liquid-solid interface on graphite. ${ }^{36-39}$

Previous work involving the STM tip-mediated removal of a physisorbed self-assembled network containing porphyrin derivatives on HOPG has been reported by Hipps et al. ${ }^{35}$ Local areas of bare graphite were obtained, however diffusion is believed to result in poorly defined structures. In contrast, the covalent linkage of aryls to the graphite surface as presented here creates a rigid platform for the formation of nanocorral patterns. Due to the localized nature of the probe and the rigidity of the grafted molecules on the surface, this nanoshaving process has the potential to create dimensionally controlled, stable corrals down to a $10 \times 10 \mathrm{~nm}^{2}$ scale.

Here, nanoshaving and subsequent liquid-solid PCDA selfassembly experiments are performed both in situ (corral creation as well as PCDA self-assembly occurs in a single step at the liquid-solid interface) and ex situ (corral creation under dry conditions followed by PCDA self-assembly at the liquid-solid interface). Corrals formed $e x$ situ show negligible impact on the PCDA domain size and shape. However, the gradual exposure of the graphite surface from in situ nanoshaving is shown to have a pronounced effect on $2 \mathrm{D}$ crystal nucleation and growth processes through the observation of fewer yet much larger domains. Using different in situ corrals, we demonstrate how nucleation and growth events involved in self-assembly can be placed under geometric and kinetic constraints to preferentially bias resultant PCDA lamellae orientation. This knowledge is used to design and experimentally demonstrate "seeding and growth" under nanoconfinement conditions.

\section{RESULTS AND DISCUSSION}

Supramolecular Self-Assembly of PCDA on Unconfined HOPG. First, supramolecular self-assembly of PCDA on freshly cleaved HOPG was evaluated as an internal benchmark 


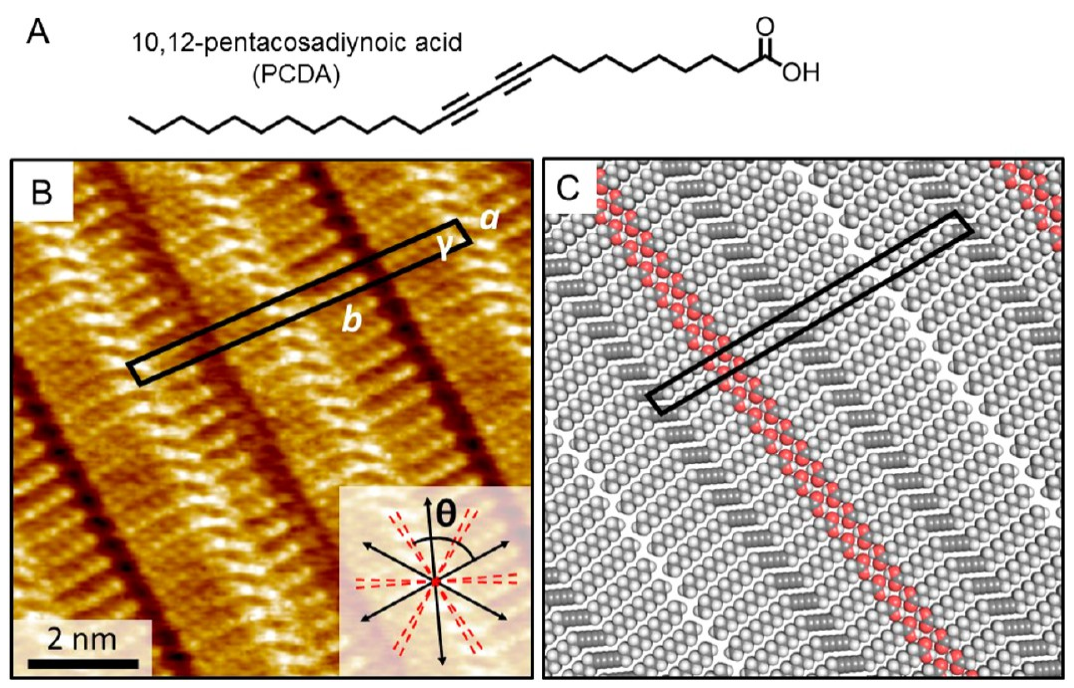

Figure 2. (a) Chemical structure of 10,12-pentacosadiynoic acid, PCDA; (b) STM image $\left(10 \times 10 \mathrm{~nm}^{2}\right)$ of PCDA self-assembled network obtained at the 1-phenyloctane/HOPG interface $\left(V_{s}=-0.8 \mathrm{~V}, I_{\mathrm{t}}=100 \mathrm{pA}\right)$. Unit cell parameters: $a=0.5 \pm 0.1 \mathrm{~nm}, b=6.6 \pm 0.1 \mathrm{~nm}, \gamma=83.8$ $\pm 0.4^{\circ}$. The inset shows the high-symmetry axes of graphite (black arrows) and the possible orientations of the lamellae (red dashed lines). The angle $\theta$ between the direction of the alkyl chains and that of the lamellae was determined to be $86.7 \pm 0.3^{\circ}$. (c) Tentative molecular model corresponding to the STM image in (b).

for comparison studies between the self-assemblies of $\mathrm{PCDA}^{40-42}$ on open terraces and laterally confined terraces. As shown in Figure 2, PCDA readily self-assembles at the 1$\mathrm{PO} / \mathrm{HOPG}$ interface. Individual molecules are clearly distinguished in the STM images. Self-assembly of the molecules is largely promoted by two types of intermolecular interactions: directional hydrogen bonding between the carboxylic acid moieties and van der Waals interactions between neighboring alkyl chains. The supramolecular packing consists of rows of parallel stacked molecules, called lamellae. The brightest features in the STM image provided in Figure $2 \mathrm{~b}$ correspond to the diacetylene units, which are tilted with respect to alkyls. The darkest rows correspond to the meeting of the methyl ends of the molecules, whereas the rows of medium contrast are assigned to the regions of hydrogen bonding. The substrate epitaxy is reflected in a selective orientation of the alkyl chains along the high-symmetry axes of graphite. The angle, $\theta$, between the lamellae direction and the graphite axis with commensurate alkyls was measured to be $86.7 \pm 0.3^{\circ}$. This results in two equivalent twinned domains for each of the three high-symmetry axes of graphite: six domain orientations in total (Figure S2).

Nanocorral Creation. To create the nanocorrals, the HOPG surface was first covalently modified with a dense monolayer of aryl species using cyclic voltammetry. The electrochemical modification of HOPG is carried out in aqueous solutions containing diazonium cations generated in situ from stable aniline precursors. ${ }^{43}$ Incorporating sterically hindering substituents on the aniline precursor limits the grafting to monolayer species. ${ }^{30,32}$ When concentrations greater than $2 \mathrm{mM}$ are used, a high density of monolayer aryl species can be covalently bound or "grafted" to the surface. After diazotization, the mechanism of covalent attachment involves reduction of the diazonium cation, expulsion of $\mathrm{N}_{2}$, and formation of aryl radicals. These unstable aryl radicals subsequently react with the HOPG surface (see Figure 1a) through a radical addition reaction. The result is a newly formed $\mathrm{sp}^{3}$-hybridized carbon-carbon bond that covalently links the aryl species to the HOPG surface. Further experimental details involving the preparation of the covalently grafted HOPG are provided in the Methods section and Supporting Information.

Nanocorrals inside the covalently modified HOPG surface are created with detailed control over the nanoshaving process using the Keysight PicoLITH 2.1 software package. Different shapes for the creation of nanocorrals are first designed within the PicoLITH software (Figure S3). The software then rasters the STM tip in the desired areas to nanoshave the corrals. The fast nanoshaving direction moves across the corral to define the width, and the slow direction moves downward to define the height of the corral. During this nanoshaving process, the STM is operating in high current (typically $200 \mathrm{pA}$ ) and low sample bias $(-1.0 \mathrm{mV})$. These scan parameters bring the tip in close proximity to the graphite substrate, such that the covalently bound aryls are degrafted and removed from the surface, i.e., nanoshaving. While the mechanism for this degrafting process is still poorly understood, the HOPG surface carbon atoms have previously been demonstrated to revert back to their original $\mathrm{sp}^{2}$ hybridization. ${ }^{30}$ Detailed investigations targeting the mechanism of nanoshaving and its dependence on nanoshaving parameters (voltage, tunneling current, and tip speed) are currently ongoing. In most cases, the tip speed for nanoshaving was set to $400 \mathrm{~nm} / \mathrm{s}$. For all of the corrals in this work, the distance covered along the slow nanoshaving direction by each raster sweep varies only slightly (between 4.0 and $8.0 \AA$ ), depending on the corral size. Thus, the time required to complete the nanoshaving depends on the area of the corral.

Assembly attempts on HOPG surfaces modified with a high density of grafted species showed no self-assembly of PCDA on top or in between the grafted features. Rather, the only observation made by STM imaging is the grafted surface itself (Figure S4). In contrast, we have recently shown that when the grafting density is lower, perturbations within the molecular self-assembly are observed. In these cases, the grafted species act as barriers and impede self-assembly. ${ }^{44}$

Ex Situ vs in Situ Creation of Nanocorrals and Its Impact on the Assembly Process. To initially establish an 


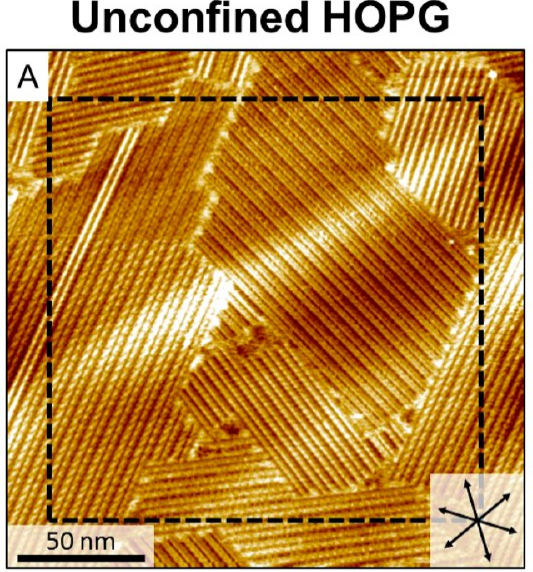

Statistical outcome

In situ nanocorral

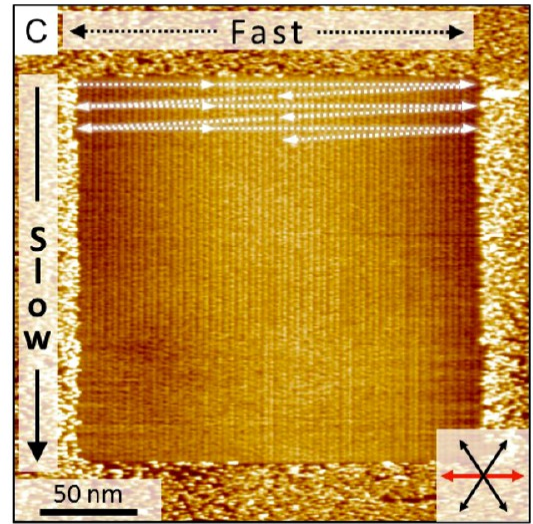

Parallel lamellae
Ex situ nanocorral

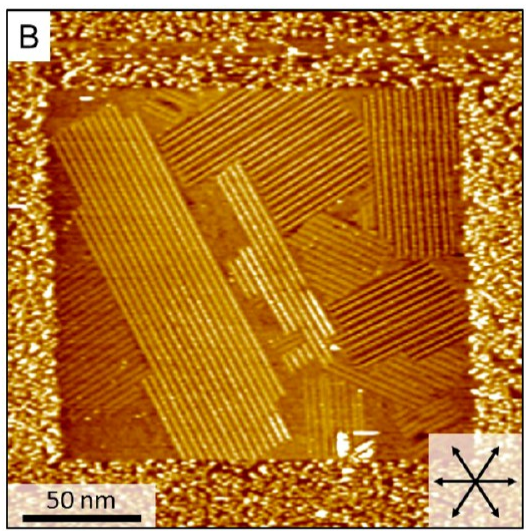

Statistical outcome

In situ nanocorral

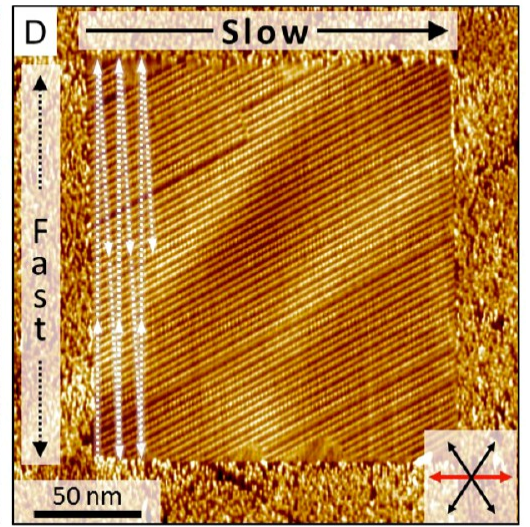

Diagonal $\left(-30^{\circ}\right)$ lamellae

Figure 3. STM images showing PCDA self-assembly (a) on freshly cleaved HOPG, (b) in an ex situ fabricated nanocorral, and (c) in an in situ nanocorral created by scanning orthogonal to a graphite symmetry axis (red arrow). Note that the lamellae are oriented parallel to the slow nanoshaving direction. (d) Within an in situ nanocorral created by scanning along one of the main symmetry axes of HOPG (red arrow). Note that the lamellae are oriented $-30^{\circ}$ with respect to the slow nanoshaving direction. Raster nanoshaving direction for corral (b) is the same as (c). The dashed square in (a) illustrates the approximate size of the nanocorrals in (b), (c), and (d). Imaging parameters for $(\mathrm{a})$ are $V_{\mathrm{s}}=-0.8$ $\mathrm{V}, I_{\mathrm{t}}=500 \mathrm{pA}$; those for (b), (c), and (d) are $V_{\mathrm{s}}=-0.8 \mathrm{~V}, I_{\mathrm{t}}=60 \mathrm{pA}$.

understanding of the influence of lateral confinement on the self-assembly of PCDA, experiments were performed on bare (unconfined) HOPG, ex situ corrals, and in situ corrals (Figure 3). Ex situ corrals were created by nanoshaving the grafted (dry) surface, then exposing the corrals to a 1-phenyloctane solution of PCDA. Within ex situ corrals, the entire nanocorral surface is exposed and self-assembly can occur in all areas. The self-assembly of PCDA inside an ex situ nanocorral $(180 \times 180$ $\mathrm{nm}^{2}$ ) was found to be nearly identical to that observed on the open terraces of unmodified HOPG. In both cases, domains of varying size and orientation are observed (Figure 3a,b). This is a strong indication that multiple nucleation events occurred. Despite the fluid environment above the corral, ripening into single domains was not observed. Similar to observations made by Beebe et al., the corral boundaries do not appear to favor nucleation, as unstable/no self-assembly is observed at the edges (Figure S5). ${ }^{21}$ Empty surface regions are also found in the assembly of PCDA on bare HOPG (Figure S5). Occasionally, features indicative of PCDA multilayer lamellae are observed inside the ex situ nanocorrals. STM topography measurements show the suspected multilayer structure is $\sim 0.5$ $\AA$ above the monolayer lamellae (Figure S6). ${ }^{45}$ Similar multilayer structures from PCDA are also observed on the bare HOPG (Figure S6). Additional STM images of the ex situ corrals and of PCDA self-assembly within ex situ nanocorrals can be found in Figures S7 and S8, respectively. From our observations it appears that assembly within relatively large $e x$ situ corrals proceeds similar to that observed on bare HOPG.

In situ corrals were created by carrying out nanoshaving directly in the presence of a 1-phenyloctane solution containing PCDA. The self-assembly of PCDA was found to be drastically different in corrals that are created in situ when compared to bare HOPG or ex situ corrals. In situ corrals almost always show a single large domain of PCDA lamellae. Importantly, the in situ created nanocorrals always show the presence of PCDA networks inside them. Thus, the self-assembly of PCDA within the in situ generated corral occurs within the time needed for the nanoshaving and the subsequent imaging scan ( $\sim 2 \mathrm{~min})$. In comparison, the time lapse between the ex situ generation of corrals and the subsequent imaging of the PCDA network was on the order of $1 \mathrm{~h}$. Thus, the assembly behavior of PCDA in the in situ nanocorrals cannot be the result of a ripening effect occurring over time. Rather, the act of nanoshaving inside the 
PCDA solution must govern the observed molecular alignment within the nanocorrals.

In Situ Nanocorral Effects: Nanoshaving Orientation with Respect to Graphite. The impact of lateral confinement on PCDA self-assembly within the in situ nanocorrals was further probed by rotating the nanoshaving orientation with respect to the underlying hexagonal graphite lattice. Importantly, the slow nanoshaving direction for the corral in Figure $3 \mathrm{c}$ is approximately orthogonal to a major axis of graphite, colored red. This creates a situation where three possible general orientations of PCDA lamellae can occur (Figure S9). Parallel, $+60^{\circ}$ diagonal, and $-60^{\circ}$ diagonal domain orientations of the PCDA lamellae can occur within the corral as defined by the angle between the direction of slow nanoshaving and the lamellae direction. The assembly within the corral appearing in Figure $3 \mathrm{c}$ is thus characterized as a parallel PCDA domain. In this case, the long axis of the molecule is parallel with the fast nanoshaving direction.

When the slow nanoshaving direction is rotated so that it runs along a major graphite axis, a different result is observed (Figure 3d). In this case, the lamellae align diagonally in the nanocorral. Assemblies that demonstrate this behavior are referred to as diagonal PCDA domains. Epitaxial matching and assembly constraints in the corral require the molecules to arrange in either a diagonal $\left(+30^{\circ}\right.$ or $\left.-30^{\circ}\right)$ or a perpendicular manner; a parallel alignment is substrate registry forbidden (Figure S9).

The assembly of PCDA displays a domain twinning effect as a result of the $2 \mathrm{D}$ chirality (Figure S2). It was not possible to selectively induce a specific $2 \mathrm{D}$ chirality within any particular nanocorral. The angle separating the equivalent mirror structures is reasoned to be too acute for such a selection. Therefore, the twinned domains are treated equally, creating only three possible categories for PCDA lamellae alignment for any specific nanoshaving direction (Figure S9). A detailed explanation of the nanoshaving process, the registry forbidden structures, and the assignment of domain behavior is further described in the Supporting Information.

For a statistical understanding of the dependence of the PCDA lamellae orientation on the nanoshaving orientation with respect to graphite, more than 50 in situ corrals of each type (orthogonal to and along a graphite major symmetry axis) were created. For faster analysis, the size of the nanocorral was reduced to $\sim 50 \times 50 \mathrm{~nm}^{2}$. When the square corrals are created with the slow nanoshaving direction orthogonal to a major graphite axis, the PCDA lamellae overwhelmingly (90.4\%) assemble into parallel PCDA domains. Compare this to the statistical outcome of $33.3 \%$. When a diagonal domain exists, they were typically accompanied by a separate parallel domain (Figure S9). Alternatively, when the slow nanoshaving direction was parallel to a major graphite axis, the reverse was observed. Diagonal PCDA lamellae are slightly preferred with a nearly equal population of + and - domains at $38.3 \%$ and $37.9 \%$, respectively. Perpendicular PCDA domains occupy only $23.8 \%$ (Figure S9). These results demonstrate that the orientation of slow nanoshaving direction with respect to the symmetry axes of graphite can be used to influence the orientation of PCDA lamellae within in situ corrals.

In Situ Nanocorral Effects: Corral Size Impact on PCDA Alignment. After establishing the difference between ex situ and in situ corrals, as well as orientational effects relative to HOPG symmetry axes, we went on to investigate the impact of the in situ nanocorral size on PCDA self-assembly. Corrals of varying sizes were created to evaluate how the nanocorral size affects the PCDA lamellae directionality bias observed from in situ nanoshaving. The nanocorrals were created by orienting the slow nanoshaving direction orthogonal to the graphite lattice, allowing the PCDA lamellae to preferentially align parallel. The results of this size-dependent study are shown in Figure 4a. In

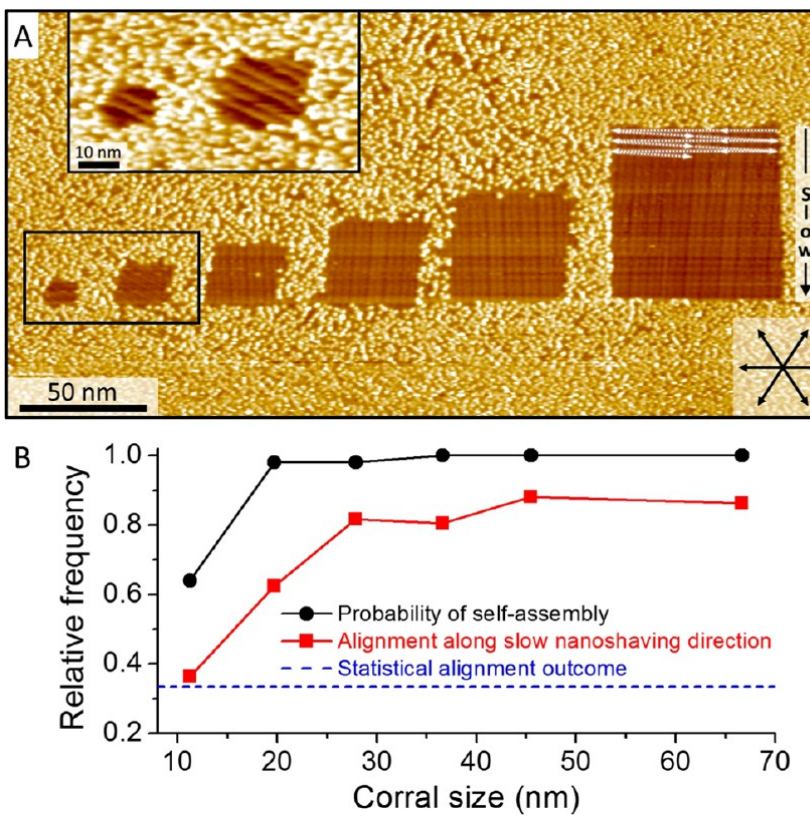

Figure 4. (a) STM image showing a series of in situ created nanocorrals with different sizes. Parallel PCDA alignment in the larger corrals and diagonal PCDA alignment in the smaller corrals (inset) are observed. Nanoshaving was performed from top to bottom for all structures shown. The high-symmetry axes of graphite are represented by black arrows. $V_{s}=-0.8 \mathrm{~V}, I_{\mathrm{t}}=60 \mathrm{pA}$. Inset: Digital zoom showing the two smaller nanocorrals. (b) Graph relating the size of the nanocorrals to the probability of selfassembly within the nanocorrals (black) and the relative frequency of PCDA domains aligned along the nanoshaving direction (red). The blue dashed line represents the statistically unbiased alignment outcome. At least $\mathbf{5 0}$ nanocorrals were analyzed for each corral size.

the four larger corrals (average size: $67 \times 67 \mathrm{~nm}^{2}, 46 \times 46 \mathrm{~nm}^{2}$, $\left.37 \times 37 \mathrm{~nm}^{2}, 28 \times 28 \mathrm{~nm}^{2}\right)$ the results are consistent with those presented in Figure 3c, where the lamellae align along the slow nanoshaving direction. For the two smaller corrals $(20 \times$ $20 \mathrm{~nm}^{2}$ and $11 \times 11 \mathrm{~nm}^{2}$ ) the PCDA lamellae are not aligned along the slow nanoshaving direction (inset Figure 4a). Instead, the lamellae are aligned diagonally with respect to the slow nanoshaving direction. A statistical representation of the dependence of lamellae orientation on the corral size is presented with a red-colored trend in Figure $4 \mathrm{~b}$. Statistics were acquired by counting the number of domains present in each corral for each particular orientation. In general, the alignment of PCDA lamellae in larger corrals is consistent until the corral size approaches $\sim 30 \mathrm{~nm}$. Below this size, the tendency of the lamellae to preferentially orient parallel decreases sharply and eventually it becomes close to the statistically unbiased outcome within corrals of $\sim 10 \mathrm{~nm}$ lateral size (Figure $4 \mathrm{~b}$ ). Thus, the orientational bias of the PCDA lamellae within in situ generated corrals is dependent on the size of the corral.

The corral size also influences the probability of self-assembly when significant confinement restraints exist (Figure 4b, black). Self-assembly is frequently observed inside square corrals of 20 

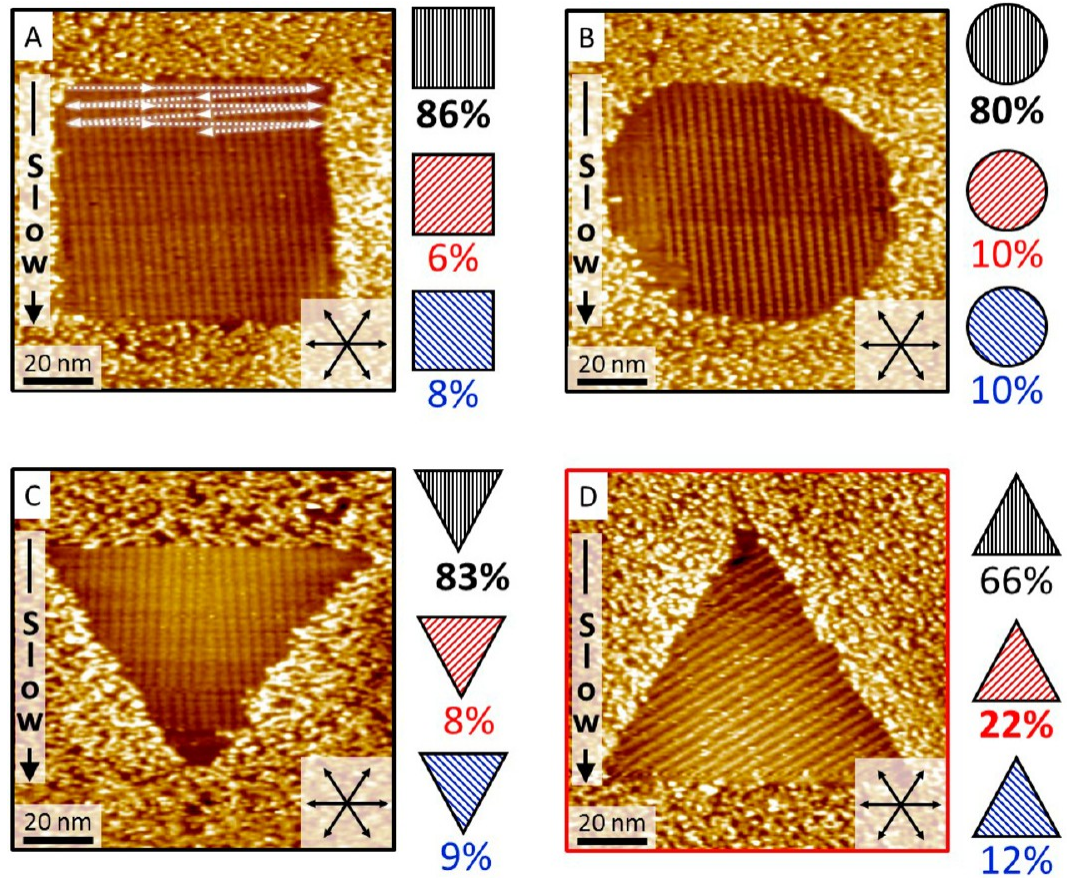

Figure 5. STM images showing PCDA self-assembly within a series of in situ corrals of different shapes: (a) square, (b) circle, (c) downward facing triangle, (d) upward facing triangle. Nanocorral height corresponds to approximately $70 \mathrm{~nm}$ for each shape. Nanoshaving direction was the same for all shapes and is indicated in the STM images. A schematic illustration of the statistical analysis of the lamellae direction inside the corrals appears alongside each image, showing parallel (black), + diagonal (red), and - diagonal (blue) orientation of the lamellae. The high-symmetry axes of graphite are represented by black arrows. (a) $-(\mathrm{d}) V_{s}=-0.8 \mathrm{~V}, I_{\mathrm{t}}=60 \mathrm{pA}$.

$\times 20 \mathrm{~nm}^{2}$ or larger. However, the propensity of the PCDA molecules to self-assemble inside corrals of $10 \times 10 \mathrm{~nm}^{2}$ is reduced to $65 \%$ (Figure $4 \mathrm{~b}$, black). A representative image of these empty corrals is shown in Figure S10. This observation is consistent with previous studies, where decreasing the size of the confined space results in prolonged molecular ordering time scales. ${ }^{21}$ It is important to note that only the image immediately after nanocorral creation was used for the analysis of the assembly statistics. Time-dependent studies under these strict nanoconfinement conditions are expected to produce other valuable insights. Nevertheless, the smallest corral created was suitable for assembly and STM imaging of an ordered PCDA domain. Corrals below $10 \mathrm{~nm}$ could not reliably be created with size and shape control. At such a small scale, the corral size begins to approach the dimension of the covalently bound aryls that confine the assembly.

In Situ Nanocorral Effects: Geometric Shape Impact on PCDA Alignment. To further investigate the impact of in situ nanocorral formation on the resultant PCDA assembly, the geometric shape of the corral was changed. Again, the slow nanoshaving direction was carefully chosen to promote parallel PCDA lamellae alignment. Nanocorrals in the shape of a square, a circle, a downward facing triangle, and an upward facing triangle are shown in Figure 5. The lamellae of PCDA align with preference along the slow nanoshaving direction for the square, the circle, and the downward triangle. A statistical analysis with more than 30 corrals of each particular shape is shown comparing the relative number of domains with a particular orientation (Figure 5). With $86 \%$ preference, the square fosters the formation of lamellae parallel to the slow nanoshaving direction. Similarly, the PCDA lamellae in circular corrals and downward triangles show a pronounced tendency to align ( $80 \%$ and $83 \%$, respectively) along the slow nanoshaving direction. The upward triangle, on the other hand, dramatically reduces this alignment trend with only $66 \%$ alignment along the slow nanoshaving direction. A (+) diagonally aligned domain inside the upright triangle, occupying $22 \%$ of the total population, is shown in Figure $5 \mathrm{~d}$. The triangle study demonstrates how the method (top-tobottom or bottom-to-top) of nanoshaving a particular shape can alter the tendency of PCDA lamellae to preferentially align. More importantly, this study demonstrates that the initial stages of the corral formation must play an important role in directing the PCDA self-assembly. Hence, the seeding and growth of the assembly of PCDA must take place as the HOPG surface is being gradually exposed.

Origin of Preferential PCDA Lamellae Alignment. The question thus arises: how does the nanoshaving of in situ nanocorrals give rise to preferential parallel PCDA lamellae alignment? Standard self-assembly of molecules at the liquidsolid interface proceeds in three distinct stages: nucleation, free growth, and ripening. ${ }^{46}$ When molecules are deposited on freshly cleaved HOPG, all of these processes contribute to the final observed structure. We propose, however, that the gradual revelation of the surface from in situ nanoshaving at the liquidsolid interface gives rise to a combination of geometric and kinetic constraints that affect the standard processes by which self-assembly typically occurs.

To demonstrate the concept of geometric constraints, we focus on the variations observed in the corral size study. Importantly, the nanoshaving of each individual corral was completed, starting with the largest corral, before moving on to the next corral. The shaving rate is consistently held at 400 $\mathrm{nm} / \mathrm{s}$. Thus, the time required for nanoshaving each particular corral decreases proportionally with decreasing size of the corral. This also creates a situation where the geometric 

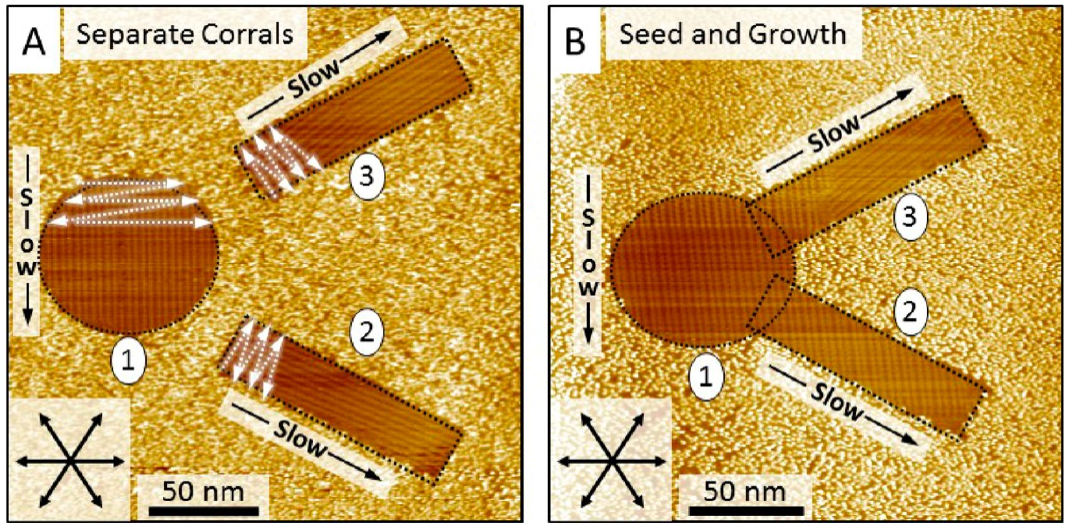

Figure 6. (a) STM image of PCDA self-assembly within a spatially separated circular corral and two additional rectangular corrals. (b) STM image of the seed and growth experiment where the circular seed corral is directly connected to the rectangular arm corrals. In this case alternative alignment is observed inside the rectangular arms. The numbers represent the order in which the structures were made. Nanoshaving direction and high-symmetry-graphite axes are indicated. $V_{\mathrm{s}}=-0.8 \mathrm{~V}, I_{\mathrm{t}}=60 \mathrm{pA}$.

limitations placed on the system at the initial stages of corral formation are very different for each particular corral. At early stages $(<2 s)$ of nanoshaving, the largest corrals exist as high aspect ratio rectangles where assembly can occur. On the other hand, the aspect ratio for the smaller nanocorrals is drastically lower for the same nanoshaving time (Figure S11). While the total nanoshaving area between the corrals is the same at these early times, the geometric constraints are very different (Figure S11). Ultimately, these different constraint conditions are believed to bias the nucleation and growth processes to impact the observed size-dependent preferential alignment.

The behavior observed in the upright and downward triangles provides strong evidence that kinetic factors created by the nanoshaving process are also influential in determining the available PCDA orientations for adsorption. The downward facing triangle is first nanoshaved along the base, whereas the upright triangle is nanoshaved from the apex first. This places the assembly of PCDA within both corrals under different kinetic constraints that limit the possible orientations for adsorption of PCDA (Figure S12). During the initial stages of corral formation, nanoshaving from the triangular base restricts the options for PCDA assembly to one particular orientation with respect to the graphite lattice (Figure S13). However, nanoshaving from the apex of the triangle opens the diagonal axes of the graphite surface at the same rate as the parallel orientation (Figure S13). Thus, the "method" of nanoshaving the triangle creates kinetic limitations that can restrict the possible PCDA assembly orientations. It is important to note that the same geometric constraints that exist within the varying sized corrals also play an important role in the triangular assembly as well. The corral size series is also influenced by similar kinetic factors that restrict assembly to one particular orientation for a period of time. Given the same nanoshaving rate, the time scales of these kinetic restrictions decrease proportionally with the size of the corral. Through these kinetic and geometric constraints, in situ nanoshaving afforded control over nucleation and growth processes to preferentially select specific orientations of the PCDA lamellae.

Finally, the presence of the potentially large STM tip in close proximity to the newly created corrals places an additional kinetic constraint on molecular adsorption events. Steric blocking effects from the tip are expected to hinder molecular diffusion in the vicinity of the tip. During this time, the PCDA molecules may experience limited access to the exposed surface.
This effect is particularly impactful when the dimensionality of the corral is similar to that of the tip. Such restrictions to PCDA self-assembly place additional kinetic constraints on the system that likely impact the observed orientation of the PCDA lamellae within small corrals (below $30 \times 30 \mathrm{~nm}^{2}$ ). Similarly, for the upward facing triangle steric hindrance of the tip might delay PCDA self-assembly within the initial degrafted area.

Assembly alignment observed in previous work from Beebe et al. inside ex situ fabricated corrals likely occurs via different pathways. ${ }^{21}$ In their work, formation of a film using a pure liquid compound resulted in assembly inside etched corrals, as well as on the open terraces of HOPG. Ripening effects and registry impacts from the assembly on the open terraces and elevated layers are believed to contribute to the observed alignment. $^{21}$ In our work, corrals of similar size to the work from Beebe et al. fabricated under dry conditions (ex situ) and later topped with a PCDA solution in 1-phenyloctane yielded multiple domain orientations. This suggests that multiple nucleation events are responsible for the final observed structure, which is not surprising given the relatively large size of the corral $\left(\sim 180 \times 180 \mathrm{~nm}^{2}\right)$ when compared to the average domain size on bare HOPG (Figure 3a). Since no assembly is observed on top of the covalently modified graphite, our assemblies cannot experience the same registry/ ripening effects from open terrace assemblies. Related to Beebe's work, we observe poor ordering at the corral boundaries, also suggesting an assembly inhibition from corral perimeters. We also never observed full molecular disassembly and reorientation within the corrals, but dynamics were occasionally observed. Beebe's methods allow for confinement studies on elevated terraces. With our corrals, such an investigation is not possible. Size, shape, and orientation control on the graphite surface can, however, be achieved. This control motivated us to probe nucleation and growth events with this highly precise method for confining molecules.

In Situ Nanocorral Effects: Seeding and Growth Demonstration. Using the constraints inherent to in situ nanocorrals, we designed an experiment to directly demonstrate the basic crystallization concept ${ }^{47,48}$ of "seeding and growth" under nanoconfinement conditions. The experiment involves the creation of a circular seed corral followed by two other rectangular arm corrals made in the presence of PCDA to allow additional growth. First, the expected alignment is shown by creating the individual corrals separately (Figure 6a). In this 
case, the alignment of the domain of PCDA lamellae inside the circular seed is parallel. Similarly, the alignment of PCDA inside the rectangular arm is also classified as parallel (given the slow nanoshaving direction), but the orientation with respect to the circular seed is clearly different. This result is expected given the nanoshaving orientation of the corrals with respect to the major graphite axes.

In a separate experiment, the rectangular arms are overlapped with the circular seed when the nanoshaving is performed. Importantly, the circular seed is nanoshaved first followed by the rectangular arms. In this case, the lamellae within the rectangular corrals are in registry with the lamellae inside the circular seed corral (Figure 6b). The PCDA lamellae are observed to run continuously from the seed into the arms. Hence, once the seed domain was formed, the PCDA growth upon exposure of additional free graphite (rectangular arm) is observed in the same direction as the lamellae observed in the seed. Occasionally, new nucleation events occur inside the arms and create domain boundaries (Figure $6 \mathrm{~b}$, top rectangle). The propensity for these new nucleation events within the rectangular corrals is expected to be dependent on the seed (and arm) size, shape, orientation, and degrafting parameters. These parameters along with the rate of surface exposure and concentration impact on 2D crystal nucleation and growth are currently under more detailed investigation.

\section{CONCLUSIONS}

In conclusion, we have revealed several general effects involved in laterally confined molecular self-assembly occurring inside nanocorrals at a liquid-solid interface. Corrals of various size, shape, and orientation are fabricated by in situ STM nanoshaving on covalently modified HOPG surfaces. Statistical analysis of these results demonstrates the importance of geometric and kinetic constraints on the resultant assembly orientation. Confinement effects during the initial stages of nanocorral formation are shown to strongly affect nucleation and growth processes. Furthermore, the confinement constraints placed on the assembly were employed for control over "seeding and growth" processes under nanoconfinement conditions. In the future coupling these corral methods with fast scanning probe microscopy techniques may allow direct observation of molecular assembly events. ${ }^{20}$ Confinement impacts on switching, chirality, multicomponent assembly, phase transitions, and stimuli-responsive networks are expected to emerge. ${ }^{1}$ The concept of nanoshaving can possibly be extended to surfaces other than HOPG, given that diazonium modification on both conducting metals and semiconducting silicon surfaces is well documented. ${ }^{34,49-55}$ The nanocorral approach and confinement principles described herein are expected to be invaluable tools for future studies on the thermodynamic and kinetic parameters involved in crystallization.

\section{METHODS}

STM Experiments. All experiments were performed at room temperature $\left(20-22{ }^{\circ} \mathrm{C}\right)$ using a PicoLE (Keysight) or Molecular Imaging STM system operating in constant-current mode at the 1phenyloctane/HOPG interface. STM tips were prepared by mechanical cutting from $\mathrm{Pt} / \mathrm{Ir}$ wire $(80 \% / 20 \%$, diameter $0.25 \mathrm{~mm})$. For self-assembly imaging, a saturated solution of 10,12-pentacosadiynoic acid $(\geq 97 \%)$ in 1-phenyloctane $(98 \%)$ was drop-casted on the surface of a freshly cleaved (or covalently modified) HOPG substrate (grade ZYB, Advanced Ceramics Inc., Cleveland, OH, USA). Both
PCDA and 1-phenyloctane were purchased from Sigma-Aldrich and used without further purification. The reported unit cell parameters of PCDA on HOPG are averaged values deduced from examination of six images that have been corrected for drift using recorded graphite images under the same conditions except for $V_{\mathrm{s}}=-1 \mathrm{mV}$ and $I_{\mathrm{t}}=200$ pA. Nanoshaving was performed using the PicoLITH v.2.1 software. All images were processed using the Scanning Probe Imaging Processor (SPIP) software (Image Metrology ApS). Imaging parameters are indicated in the figure captions and are denoted by $V_{\mathrm{s}}$ for the sample bias and $I_{\mathrm{t}}$ for the tunneling current.

Covalent Modification of HOPG. Electrochemical grafting of 3,5bis-tert-butylbenzenediazonium (3,5-TBD) was performed using cyclic voltammetry in aqueous solutions. Due to the low stability of 3,5-TBD, it was generated in situ from the corresponding aniline compound. To this end, $\geq 3$ mg of 3,5-bis-tert-butylaniline (3,5-TBA) was dissolved in $5 \mathrm{~mL}$ of aqueous hydrochloric acid $(50 \mathrm{mM})$, and $100 \mu \mathrm{L}$ of aqueous $\mathrm{NaNO}_{2}(0.1 \mathrm{M})$ was added for activation of the diazotization reaction. The solution was gently shaken for $1.5 \mathrm{~min}$ before injection into the electrochemical cell. A lab-built single-compartment three-electrode cell, with a working electrode area of $50.3 \mathrm{~mm}^{2}$, Pt wire counter, and $\mathrm{Ag} / \mathrm{AgCl} / 3.0 \mathrm{M} \mathrm{NaCl}$ reference electrode was used to carry out the cyclic voltammetry. Prior to each experiment, the HOPG working electrode was freshly cleaved using Scotch tape. A typical currentvoltage diagram obtained during cyclic voltammetry is shown in Figure S1. Every experiment consisted of three voltage sweeps. After modification, the HOPG samples were rinsed with Milli-Q water to remove any physisorbed material from the surface. 3,5-Bis-tertbutylaniline (98\%) and analytical grade hydrochloric acid were purchased from Sigma-Aldrich and used without further purification. High-purity water (Milli- $\mathrm{Q}$, Millipore, $18.2 \mathrm{M} \Omega \mathrm{cm}$, TOC $<3 \mathrm{ppb}$ ) was used for preparation of the aqueous solutions. All electrochemical measurements were performed using an Autolab PGSTAT101 potentiostat (Metrohm_Autolab BV, The Netherlands).

\section{ASSOCIATED CONTENT}

\section{S Supporting Information}

The Supporting Information is available free of charge on the ACS Publications website at DOI: 10.1021/acsnano.6b05954.

Extra STM and cyclic voltammetry results; schematics illustrating geometric and kinetic constraints during in situ self-assembly (PDF)

\section{AUTHOR INFORMATION}

\section{Corresponding Authors}

*E-mail: john.greenwood@nottingham.ac.uk.

*E-mail: steven.defeyter@kuleuven.be.

\section{Present Address}

${ }^{\dagger}$ School of Physics \& Astronomy, University of Nottingham, Nottingham, NG7 2RD, United Kingdom.

\section{Notes}

The authors declare no competing financial interest.

\section{ACKNOWLEDGMENTS}

This work has received funding from the Fund of Scientific Research-Flanders (FWO), KU Leuven Internal Funds, Belgian Federal Science Policy Office (IAP-7/05), and the European Research Council under the European Union's Seventh Framework Programme (FP7/2007-2013)/ERC Grant Agreement No. 340324. L.V. thanks the FWO for the fellowship received. B.H. acknowledges the Belgian American Educational Foundation and the FWO.

\section{REFERENCES}

(1) Mali, K. S.; Adisoejoso, J.; Ghijsens, E.; De Cat, I.; De Feyter, S. Exploring the Complexity of Supramolecular Interactions for 
Patterning at the Liquid-Solid Interface. Acc. Chem. Res. 2012, 45, $1309-1320$.

(2) Lackinger, M.; Griessl, S.; Heckl, W. M.; Hietschold, M.; Flynn, G. W. Self-Assembly of Trimesic Acid at the Liquid-Solid Interface - a Study of Solvent-Induced Polymorphism. Langmuir 2005, 21, 49844988.

(3) Blunt, M. O.; Russell, J. C.; Giménez-López, M. d. C.; Garrahan, J. P.; Lin, X.; Schröder, M.; Champness, N. R.; Beton, P. H. Random Tiling and Topological Defects in a Two-Dimensional Molecular Network. Science 2008, 322, 1077-1081.

(4) Xue, Y.; Zimmt, M. B. Patterned Monolayer Self-Assembly Programmed by Side Chain Shape: Four-Component Gratings. J. Am. Chem. Soc. 2012, 134, 4513-4516.

(5) Cahen, D.; Kahn, A.; Umbach, E. Energetics of Molecular Interfaces. Mater. Today 2005, 8, 32-41.

(6) Huang, H.; Chen, S.; Gao, X.; Chen, W.; Wee, A. T. S. Structural and Electronic Properties of PTCDA Thin Films on Epitaxial Graphene. ACS Nano 2009, 3, 3431-3436.

(7) Gates, B. D.; Xu, Q.; Stewart, M.; Ryan, D.; Willson, C. G.; Whitesides, G. M. New Approaches to Nanofabrication: Molding, Printing, and Other Techniques. Chem. Rev. 2005, 105, 1171-1196.

(8) Wang, Q. H.; Hersam, M. C. Room-Temperature MolecularResolution Characterization of Self-Assembled Organic Monolayers on Epitaxial Graphene. Nat. Chem. 2009, 1, 206-211.

(9) Zhang, J. L.; Zhong, S.; Zhong, J. Q.; Niu, T. C.; Hu, W. P.; Wee, A. T. S.; Chen, W. Rational Design of Two-Dimensional Molecular Donor-Acceptor Nanostructure Arrays. Nanoscale 2015, 7, 43064324.

(10) Lackinger, M.; Griessl, S.; Markert, T.; Jamitzky, F.; Heckl, W. M. Self-Assembly of Benzene-Dicarboxylic Acid Isomers at the Liquid Solid Interface: Steric Aspects of Hydrogen Bonding. J. Phys. Chem. B 2004, 108, 13652-13655.

(11) Blunt, M. O.; Adisoejoso, J.; Tahara, K.; Katayama, K.; Van der Auweraer, M.; Tobe, Y.; De Feyter, S. Temperature-Induced Structural Phase Transitions in a Two-Dimensional Self-Assembled Network. J. Am. Chem. Soc. 2013, 135, 12068-12075.

(12) Marie, C.; Silly, F.; Tortech, L.; Müllen, K.; Fichou, D. Tuning the Packing Density of 2D Supramolecular Self-Assemblies at the Solid-Liquid Interface Using Variable Temperature. ACS Nano 2010, 4, 1288-1292.

(13) Silly, F. Two-Dimensional 1,3,5-Tris(4-Carboxyphenyl)Benzene Self-Assembly at the 1-Phenyloctane/Graphite Interface Revisited. J. Phys. Chem. C 2012, 116, 10029-10032.

(14) Mamdouh, W.; Uji-i, H.; Ladislaw, J. S.; Dulcey, A. E.; Percec, V.; De Schryver, F. C.; De Feyter, S. Solvent Controlled Self-Assembly at the Liquid-Solid Interface Revealed by STM. J. Am. Chem. Soc. 2006, $128,317-325$.

(15) Zhang, X.; Chen, T.; Chen, Q.; Deng, G.-J.; Fan, Q.-H.; Wan, L.-J. One Solvent Induces a Series of Structural Transitions in Monodendron Molecular Self-Assembly from Lamellar to Quadrangular to Hexagonal. Chem. - Eur. J. 2009, 15, 9669-9673.

(16) Lee, S.-L.; Chi, C.-Y. J.; Huang, M.-J.; Chen, C.-h.; Li, C.-W.; Pati, K.; Liu, R.-S. Shear-Induced Long-Range Uniaxial Assembly of Polyaromatic Monolayers at Molecular Resolution. J. Am. Chem. Soc. 2008, 130, 10454-10455.

(17) Balandina, T.; Tahara, K.; Sändig, N.; Blunt, M. O.; Adisoejoso, J.; Lei, S.; Zerbetto, F.; Tobe, Y.; De Feyter, S. Role of Substrate in Directing the Self-Assembly of Multicomponent Supramolecular Networks at the Liquid-Solid Interface. ACS Nano 2012, 6, 83818389.

(18) Song, W.; Martsinovich, N.; Heckl, W. M.; Lackinger, M. Thermodynamics of Halogen Bonded Monolayer Self-Assembly at the Liquid-Solid Interface. Chem. Commun. 2014, 50, 13465-13468.

(19) Mazur, U.; Hipps, K. W. Kinetic and Thermodynamic Processes of Organic Species at the Solution-Solid Interface: The View through an STM. Chem. Commun. 2015, 51, 4737-4749.

(20) Weigelt, S.; Busse, C.; Petersen, L.; Rauls, E.; Hammer, B.; Gothelf, K. V.; Besenbacher, F.; Linderoth, T. R. Chiral Switching by
Spontaneous Conformational Change in Adsorbed Organic Molecules. Nat. Mater. 2006, 5, 112-117.

(21) Patrick, D. L.; Cee, V. J.; Beebe, T. P. Molecule Corrals for Studies of Monolayer Organic Films. Science 1994, 265, 231-234.

(22) Zhu, Y. J.; McBride, J. D.; Hansen, T. A.; Beebe, T. P. Controlled Production of Molecule Corrals Using Cesium Ion Bombardment: A TOF-SIMS, XPS, and STM Study. J. Phys. Chem. B 2001, 105, 2010-2018.

(23) Zhu, Y. J.; Hansen, T. A.; Ammermann, S.; McBride, J. D.; Beebe, T. P. Nanometer-Size Monolayer and Multilayer Molecule Corrals on HOPG: A Depth-Resolved Mechanistic Study by STM. J. Phys. Chem. B 2001, 105, 7632-7638.

(24) de Oteyza, D. G.; Barrena, E.; Dosch, H.; Wakayama, Y. Nanoconfinement Effects in the Self-Assembly of Diindenoperylene (DIP) on $\mathrm{Cu}(111)$ Surfaces. Phys. Chem. Chem. Phys. 2009, 11, 87418744.

(25) Cirera, B.; Zhang, Y.-Q.; Björk, J.; Klyatskaya, S.; Chen, Z.; Ruben, M.; Barth, J. V.; Klappenberger, F. Synthesis of Extended Graphdiyne Wires by Vicinal Surface Templating. Nano Lett. 2014, 14, 1891-1897.

(26) Fan, Q. T.; Dai, J. Y.; Wang, T.; Kuttner, J.; Hilt, G.; Gottfried, J. M.; Zhu, J. F. Confined Synthesis of Organometallic Chains and Macrocycles by $\mathrm{Cu}-\mathrm{O}$ Surface Templating. ACS Nano 2016, 10, 3747-3754.

(27) Crommie, M. F.; Lutz, C. P.; Eigler, D. M. Confinement of Electrons to Quantum Corrals on a Metal Surface. Science 1993, 262, 218-220.

(28) Cheng, Z.; Wyrick, J.; Luo, M.; Sun, D.; Kim, D.; Zhu, Y.; Lu, W.; Kim, K.; Einstein, T. L.; Bartels, L. Adsorbates in a Box: Titration of Substrate Electronic States. Phys. Rev. Lett. 2010, 105, 066104.

(29) Pawin, G.; Wong, K. L.; Kwon, K.-Y.; Bartels, L. A Homomolecular Porous Network at a $\mathrm{Cu}(111)$. Science 2006, 313, 961-962.

(30) Greenwood, J.; Phan, T. H.; Fujita, Y.; Li, Z.; Ivasenko, O.; Vanderlinden, W.; Van Gorp, H.; Frederickx, W.; Lu, G.; Tahara, K.; Tobe, Y.; Uji-i, H.; Mertens, S. F. L.; De Feyter, S. Covalent Modification of Graphene and Graphite Using Diazonium Chemistry: Tunable Grafting and Nanomanipulation. ACS Nano 2015, 9, 55205535.

(31) Downard, A. J. Electrochemically Assisted Covalent Modification of Carbon Electrodes. Electroanalysis 2000, 12, 1085-1096.

(32) Combellas, C.; Kanoufi, F.; Pinson, J.; Podvorica, F. I. Sterically Hindered Diazonium Salts for the Grafting of a Monolayer on Metals. J. Am. Chem. Soc. 2008, 130, 8576-8577.

(33) Lee, L.; Brooksby, P. A.; Hapiot, P.; Downard, A. J. Electrografting of 4-Nitrobenzenediazonium Ion at Carbon Electrodes: Catalyzed and Uncatalyzed Reduction Processes. Langmuir 2016, 32, $468-476$.

(34) Belanger, D.; Pinson, J. Electrografting: A Powerful Method for Surface Modification. Chem. Soc. Rev. 2011, 40, 3995-4048.

(35) Scudiero, L.; Hipps, K. W. Controlled Manipulation of SelfOrganized $\mathrm{Ni}(\mathrm{II})$-Octaethylporphyrin Molecules Deposited from Solution on HOPG with a Scanning Tunneling Microscope. J. Phys. Chem. C 2007, 111, 17516-17520.

(36) MacLeod, J. M.; Ivasenko, O.; Fu, C. Y.; Taerum, T.; Rosei, F.; Perepichka, D. F. Supramolecular Ordering in OligothiopheneFullerene Monolayers. J. Am. Chem. Soc. 2009, 131, 16844-16850.

(37) Lackinger, M.; Heckl, W. M. Carboxylic Acids: Versatile Building Blocks and Mediators for Two-Dimensional Supramolecular Self-Assembly. Langmuir 2009, 25, 11307-11321.

(38) Dickerson, P. N.; Hibberd, A. M.; Oncel, N.; Bernasek, S. L. Hydrogen-Bonding versus van der Waals Interactions in SelfAssembled Monolayers of Substituted Isophthalic Acids. Langmuir 2010, 26, 18155-18161.

(39) Park, K. W.; Adisoejoso, J.; Plas, J.; Hong, J.; Mullen, K.; De Feyter, S. Self-Assembly Behavior of Alkylated Isophthalic Acids Revisited: Concentration in Control and Guest-Induced Phase Transformation. Langmuir 2014, 30, 15206-15211. 
(40) Okawa, Y.; Aono, M. Linear Chain Polymerization Initiated by a Scanning Tunneling Microscope Tip at Designated Positions. J. Chem. Phys. 2001, 115, 2317-2322.

(41) Giridharagopal, R.; Kelly, K. F. Substrate-Dependent Properties of Polydiacetylene Nanowires on Graphite and MoS2. ACS Nano 2008, 2, 1571-1580.

(42) Deshpande, A.; Sham, C. H.; Alaboson, J. M. P.; Mullin, J. M.; Schatz, G. C.; Hersam, M. C. Self-Assembly and Photopolymerization of Sub-2 nm One-Dimensional Organic Nanostructures on Graphene. J. Am. Chem. Soc. 2012, 134, 16759-16764.

(43) Cougnon, C.; Nguyen, N. H.; Dabos-Seignon, S.; Mauzeroll, J.; Bélanger, D. Carbon Surface Derivatization by Electrochemical Reduction of a Diazonium Salt in situ Produced from the Nitro Precursor. J. Electroanal. Chem. 2011, 661, 13-19.

(44) Braganca, A. M.; Greenwood, J.; Ivasenko, O.; Phan, T. H.; Mullen, K.; De Feyter, S. The Impact of Grafted Surface Defects and Their Controlled Removal on Supramolecular Self-Assembly. Chem. Sci. 2016, DOI: 10.1039/C6SC02400A.

(45) Gilbert, E. P.; White, J. W.; Senden, T. J. Evidence for Perpendicular n-Alkane Orientation at the Liquid/Graphite Interface. Chem. Phys. Lett. 1994, 227, 443-446.

(46) Kim, K.; Plass, K. E.; Matzger, A. J. Kinetic and Thermodynamic Forms of a Two-Dimensional Crystal. Langmuir 2003, 19, 7149-7152.

(47) Belcher, A. M.; Wu, X. H.; Christensen, R. J.; Hansma, P. K.; Stucky, G. D.; Morse, D. E. Control of Crystal Phase Switching and Orientation by Soluble Mollusc-Shell Proteins. Nature 1996, 381, 5658.

(48) Aizenberg, J.; Black, A. J.; Whitesides, G. M. Control of Crystal Nucleation by Patterned Self-Assembled Monolayers. Nature 1999, 398, 495-498.

(49) Pinson, J.; Podvorica, F. Attachment of Organic Layers to Conductive or Semiconductive Surfaces by Reduction of Diazonium Salts. Chem. Soc. Rev. 2005, 34, 429-439.

(50) Lehr, J.; Garrett, D. J.; Paulik, M. G.; Flavel, B. S.; Brooksby, P. A.; Williamson, B. E.; Downard, A. J. Patterning of Metal, Carbon, and Semiconductor Substrates with Thin Organic Films by Microcontact Printing with Aryldiazonium Salt Inks. Anal. Chem. 2010, 82, 70277034.

(51) Laforgue, A.; Addou, T.; Belanger, D. Characterization of the Deposition of Organic Molecules at the Surface of Gold by the Electrochemical Reduction of Aryldiazonium Cations. Langmuir 2005, 21, 6855-6865.

(52) Liu, G. Z.; Bocking, T.; Gooding, J. J. Diazonium Salts: Stable Monolayers on Gold Electrodes for Sensing Applications. J. Electroanal. Chem. 2007, 600, 335-344.

(53) Laurentius, L.; Stoyanov, S. R.; Gusarov, S.; Kovalenko, A.; Du, R. B.; Lopinski, G. P.; McDermott, M. T. Diazonium-Derived Aryl Films on Gold Nanoparticles: Evidence for a Carbon-Gold Covalent Bond. ACS Nano 2011, 5, 4219-4227.

(54) Shewchuk, D. M.; McDermott, M. T. Comparison of Diazonium Salt Derived and Thiol Derived Nitrobenzene Layers on Gold. Langmuir 2009, 25, 4556-4563.

(55) Lehr, J.; Williamson, B. E.; Flavel, B. S.; Downard, A. J. Reaction of Gold Substrates with Diazonium Salts in Acidic Solution at OpenCircuit Potential. Langmuir 2009, 25, 13503-13509. 


\section{Supporting Information}

\section{Self-Assembly under Confinement: Nanocorrals for Understanding Fundamentals of 2D Crystallization}

Lander Verstraete, John Greenwood, Brandon E. Hirsch, Steven De Feyter

Department of Chemistry, Division of Molecular Imaging and Photonics, KU LeuvenUniversity of Leuven, Celestijnenlaan 200F, B-3001 Leuven, Belgium.

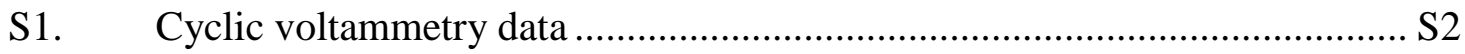

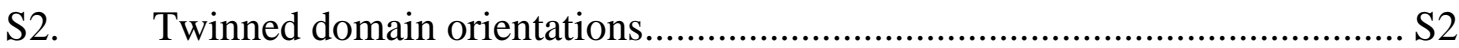

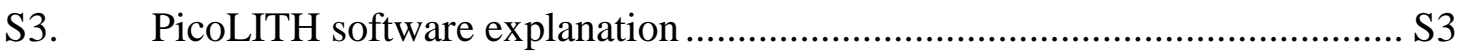

S4. No PCDA assembly on top of grafted structures ........................................ S4

S5. Bare HOPG areas in unconfined HOPG, ex situ, and in situ corrals ............. S5

S6. Multilayer observations in unconfined HOPG and ex situ corrals................ S6

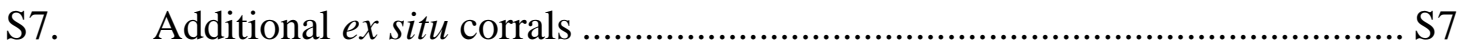

S8. In situ nanoshaving orientational dependence on lamellae alignment........... S8

S9. Bare graphite observation in small in situ corrals .................................. S10

S10. Geometric constraints within in situ nanocorral size series ........................ S11

S11. Kinetic constraints involved in the triangular corrals .............................. S12 


\section{S1. Cyclic voltammetry data}

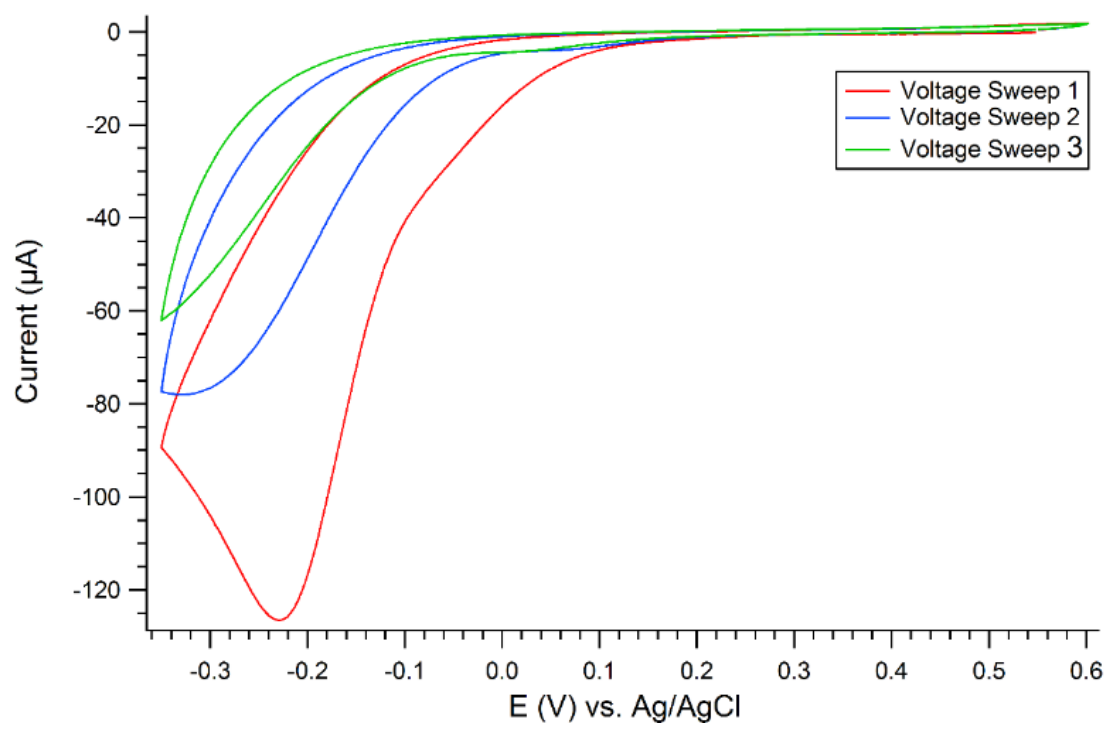

Figure S1. Cyclic voltammetry of HOPG in $2 \mathrm{mM} 3,5-\mathrm{TBD}$. Potential limits: 0.6 to $-0.35 \mathrm{~V}$. The irreversible current peak at around $-0.23 \mathrm{~V} v s$. $\mathrm{Ag} / \mathrm{AgCl}$ is assigned to the reduction of 3,5$\mathrm{TBD}$, forming the corresponding radicals that appear in Figure 1a.

\section{S2. Twinned domain orientations}

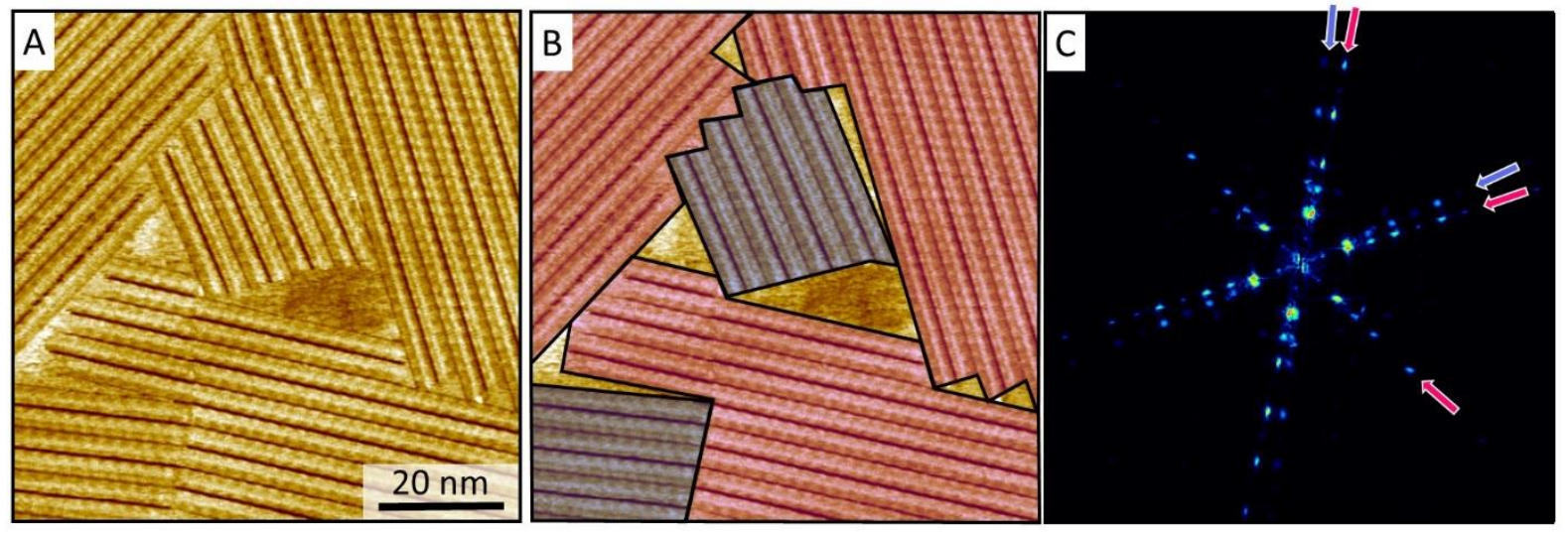

Figure S2. (a) STM image (100 x $\left.100 \mathrm{~nm}^{2}\right)$ showing the twinning of PCDA lamellae domains. $\left(V_{s}=-0.7 \mathrm{~V}, I_{t}=300 \mathrm{pA}\right.$ ) (b) STM image color overlays, red and blue, show the difference between each twinned domain with respect to the graphite. (c) Corresponding fast Fourier transform (FFT) to the image in (a), with the twinning of the lamellae orientations. 


\section{S3. PicoLITH software explanation}

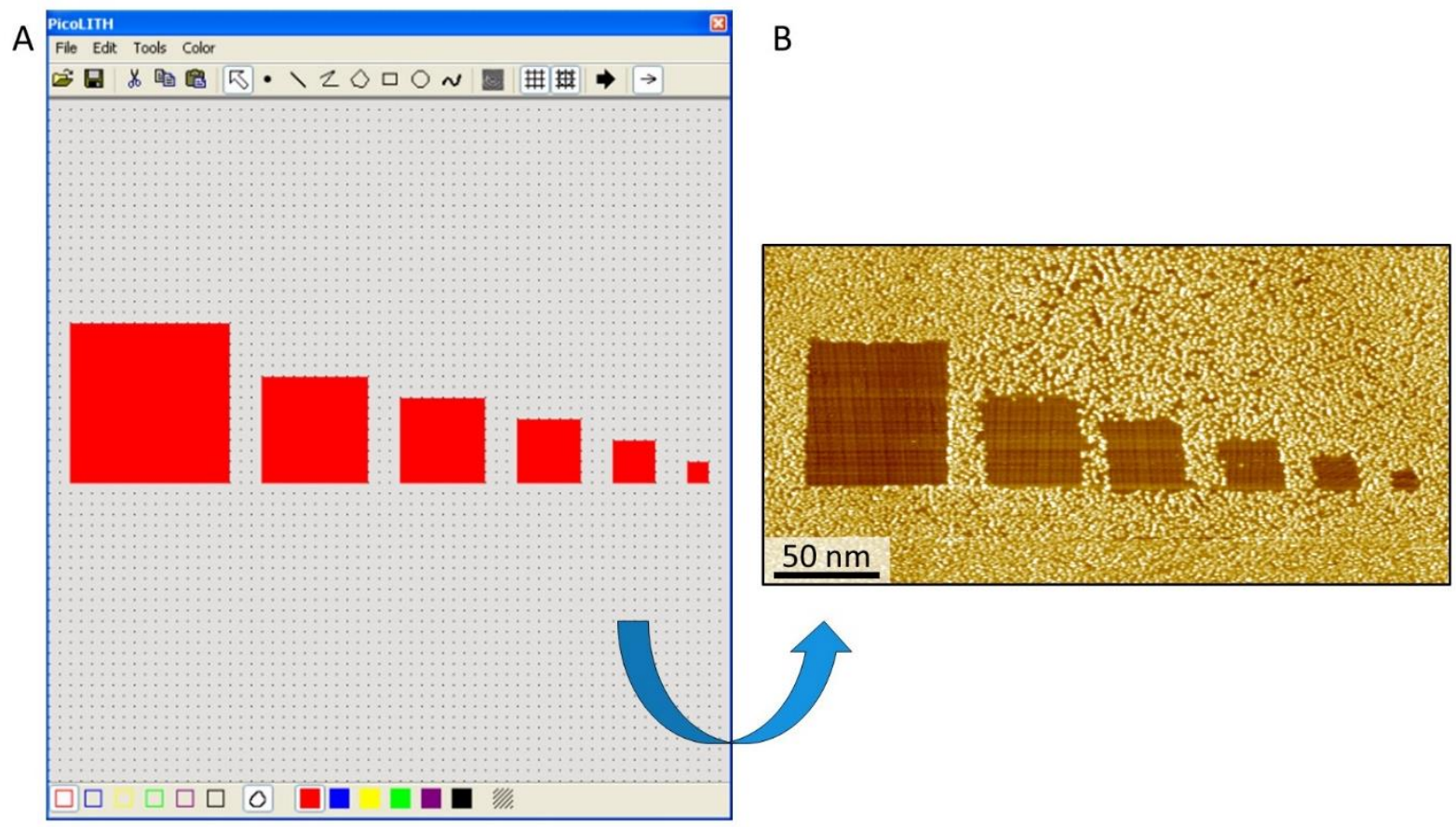

Figure S3. (a) Corrals are designed in the PicoLITH software, and then subsequently nanoshaved into the densely grafted HOPG surface. (b) STM image acquired immediately after the nanoshaving of the pattern designed in (a). In this particular image, the template in the PicoLITH software is set at $320 \times 320 \mathrm{~nm}^{2}$ total area. The dot grid displays a spacing distance of $5 \mathrm{~nm}$. STM image recorded with the parameters $V_{s}=-0.8 \mathrm{~V}, I_{t}=60 \mathrm{pA}$. Importantly, the nanoshaving process was carried out with $V_{s}=-0.001 \mathrm{~V}, I_{t}=200 \mathrm{pA}$. From largest to smallest corral size, the total time take for nanoshaving is $34.7 \mathrm{sec}, 16.1 \mathrm{sec}, 10.5 \mathrm{sec}, 6.1 \mathrm{sec}, 3.0 \mathrm{sec}$, $1.0 \mathrm{sec}$. It is also important to note that each square corral is completed before moving onto the next box. In other words, the tip does not scan the full line profile of the pattern but rather each object individually. 


\section{S4. No PCDA assembly on top of grafted structures}
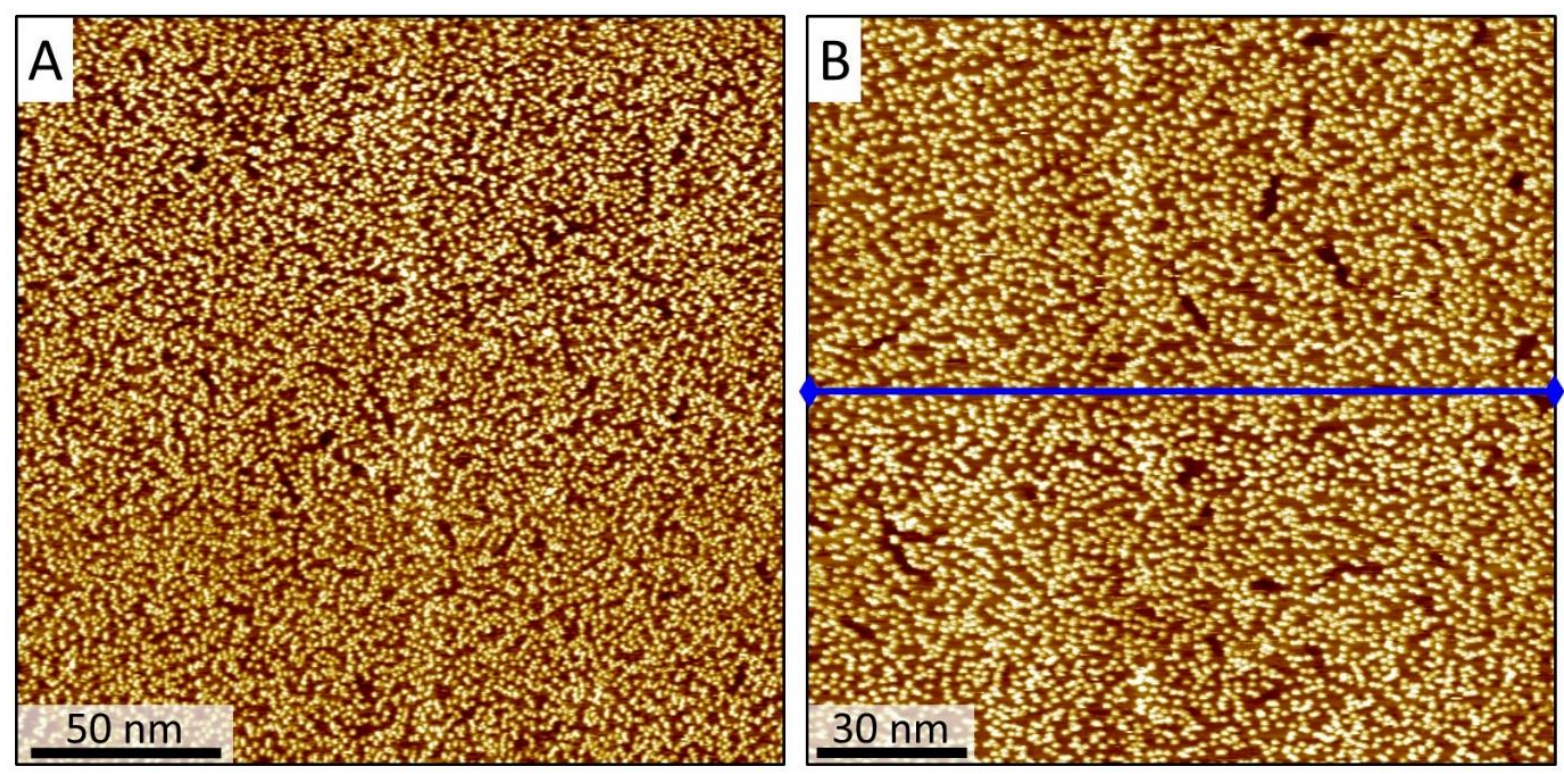

C

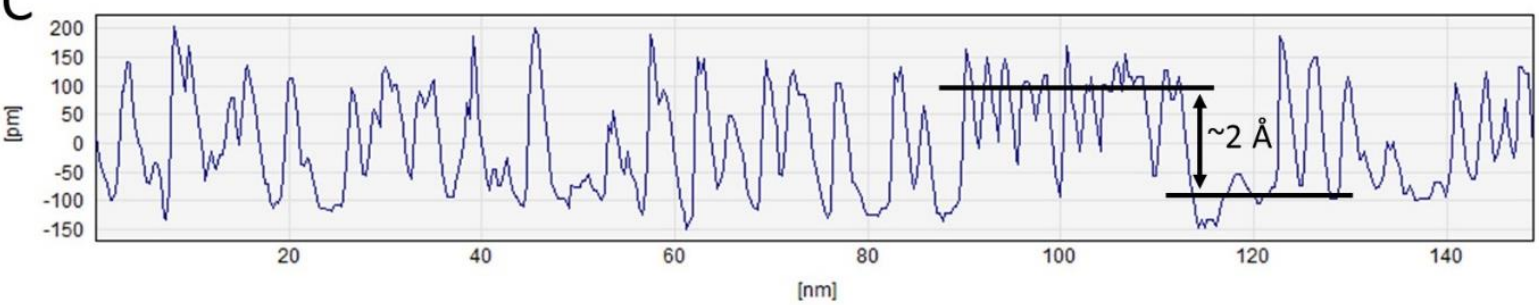

Figure S4. (a \& b) STM images of the covalently modified HOPG surface. Bright features in the images are assigned to the covalently bound aryl species. Both images are acquired at the liquid-solid interface with a saturated solution of PCDA in 1-phenyloctane. No indications of PCDA assembly can be found on the surface. Image parameters for (a) are $V_{s}=-0.8 \mathrm{~V}, I_{t}=60$ $\mathrm{pA}$ and for (b) are $V_{s}=-0.8 \mathrm{~V}, I_{t}=100 \mathrm{pA}$. (c) STM apparent height line profile across image (b). The height difference between the covalently bound aryl species and the bare graphite surface measures $\sim 2 \AA$. 
S5. Bare HOPG areas in unconfined HOPG, ex situ, and in situ corrals
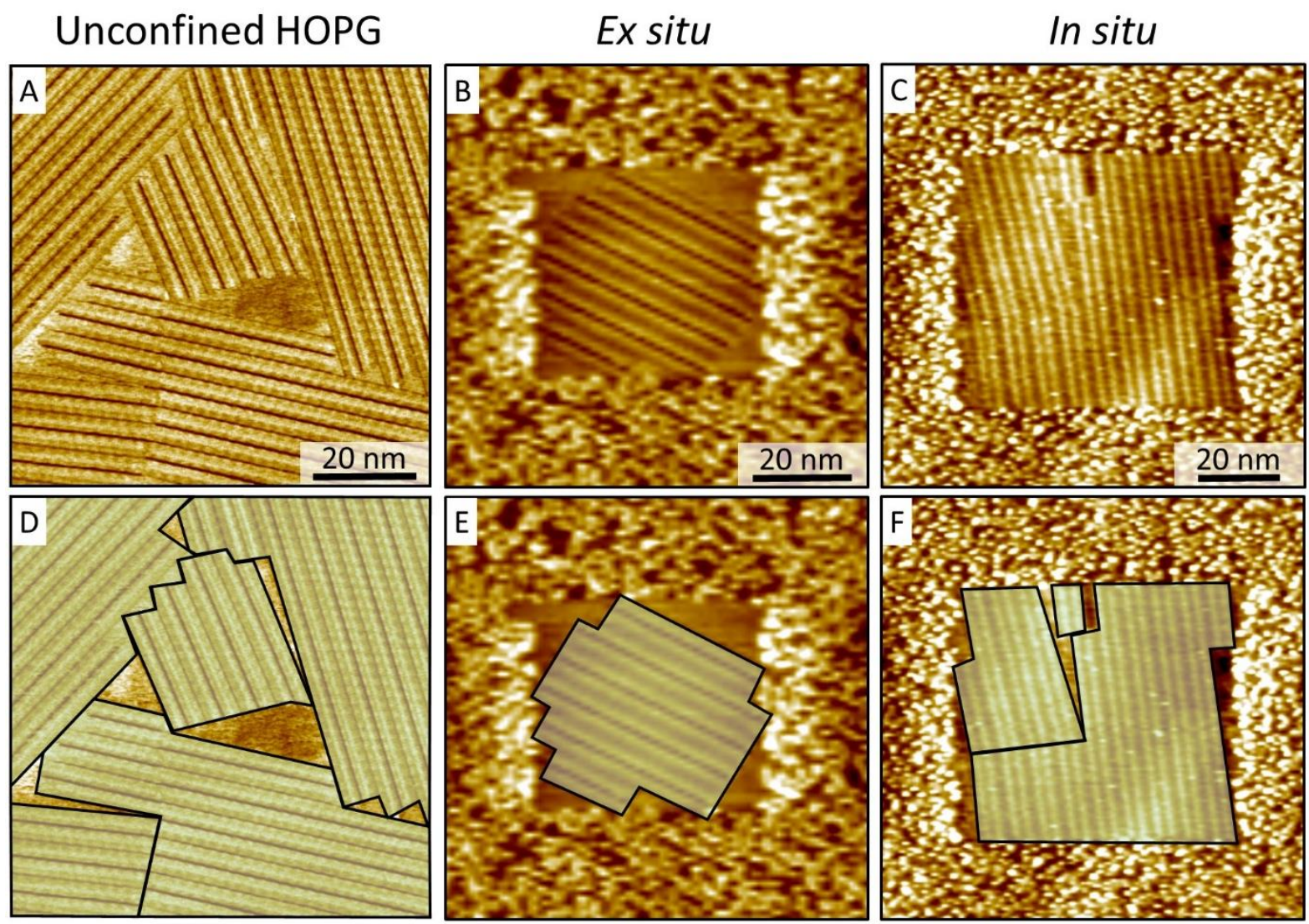

Figure S5. Comparison on PCDA assemblies in different conditions: unconfined HOPG (a), ex situ nanocorral (b), and in situ nanocorral (c). In all case bare areas on the graphite surface are observed. The PCDA lamellae are masked in (d), (e), and (f) to highlight the bare HOPG areas. Image parameters (a) $V_{s}=-0.7 \mathrm{~V}, I_{t}=300 \mathrm{pA}$, (b) $V_{s}=-0.8 \mathrm{~V}, I_{t}=60 \mathrm{pA}$, (c). $V_{s}=-0.8$ $\mathrm{V}, I_{t}=60 \mathrm{pA}$. 
S6. Multilayer observations in unconfined HOPG and ex situ corrals
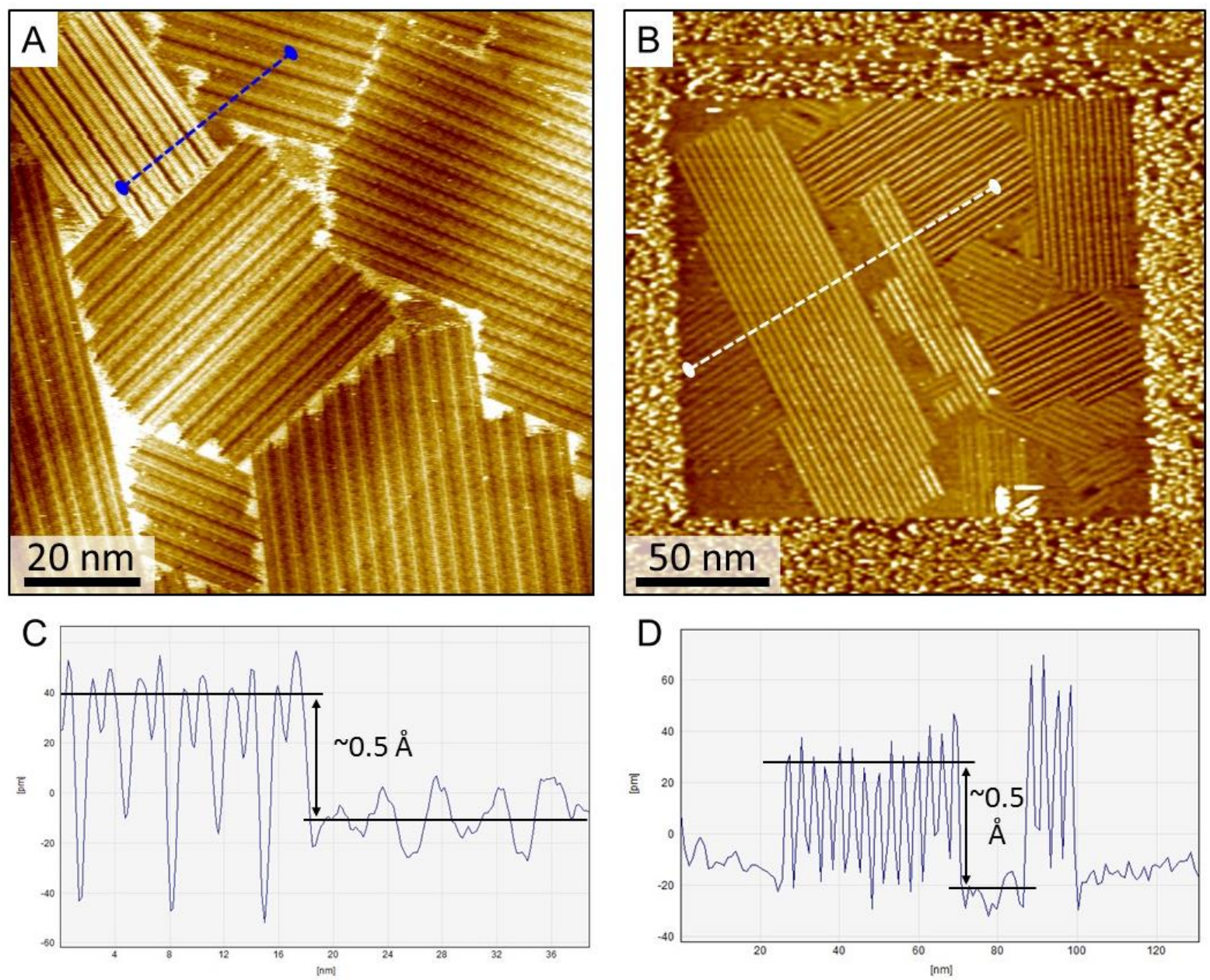

Figure S6. Comparison on multilayer observation of PCDA assemblies (a) for unconfined HOPG and (b) for corrals created ex situ. STM apparent height line profiles from image (a) appears in (c) and similarly the line profile in (b) appears in (d). The STM topography distance between the lamella is $\sim 0.5 \AA$ on average. Image parameters (a) $V_{s}=-0.7 \mathrm{~V}, I_{t}=300 \mathrm{pA}$; (b) $V_{s}=-0.8 \mathrm{~V}, I_{t}=60 \mathrm{pA}$. 


\section{S7. Additional ex situ corrals}
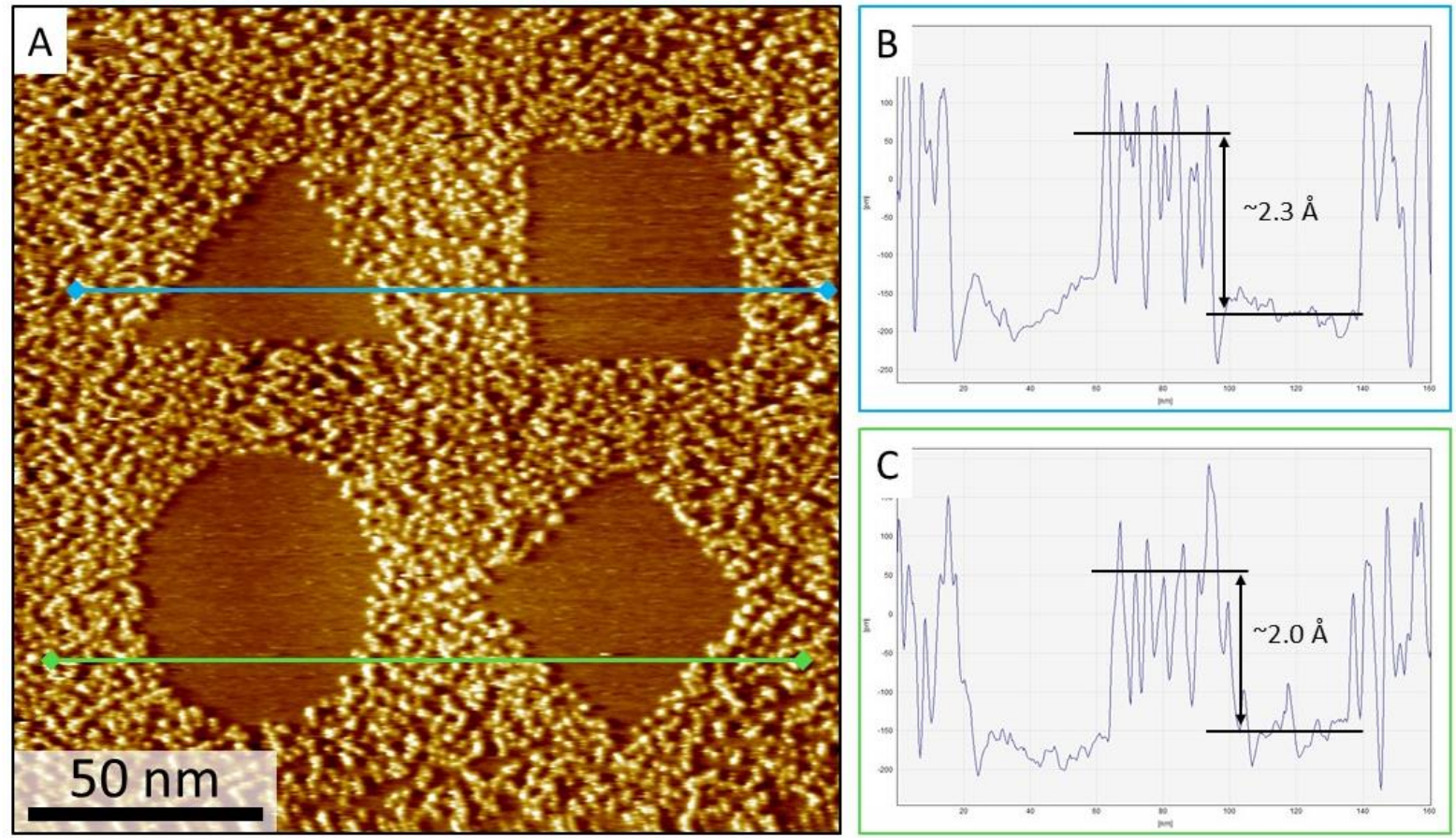

Figure S7. (a) STM image of ex situ created nanocorrals obtained under dry conditions. Image parameters are $V_{s}=-0.8 \mathrm{~V}, I_{t}=60 \mathrm{pA}$. (b \& c) Corresponding STM apparent height line profiles from image (a) demonstrating the STM topography change between the covalently modified surface and the empty corrals.
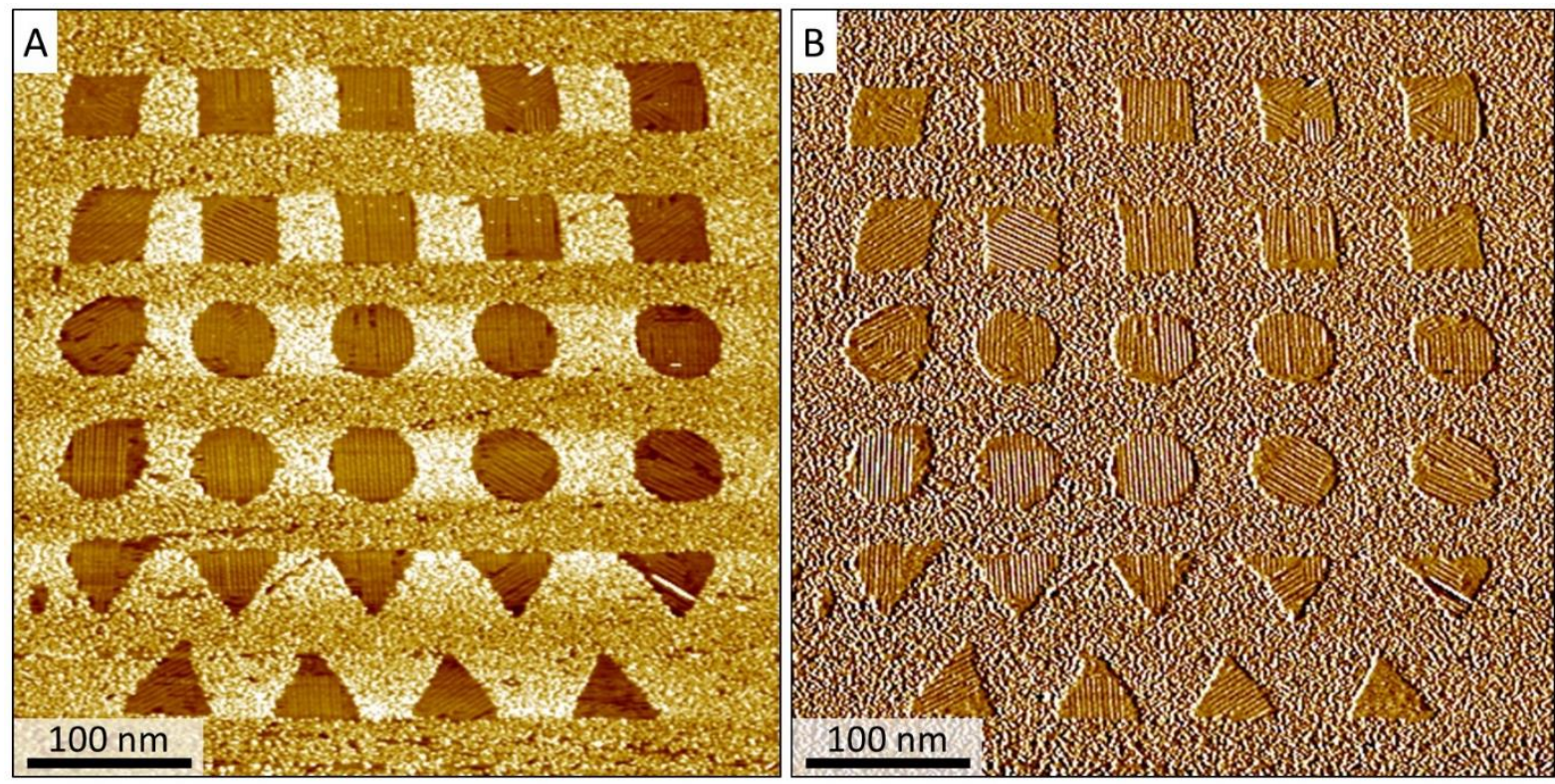

Figure S8. STM image of PCDA self-assembly within an array of different shaped ex situ nanocorrals on HOPG. (a) Topography image, and (b) corresponding current image. $V_{s}=-0.8$ $\mathrm{V}, I_{t}=60 \mathrm{pA}$. PCDA was dropcasted from a saturated 1-phenyloctane solution after the corrals were nanoshaved under dry conditions. 
S8. In situ nanoshaving orientational dependence on lamellae alignment

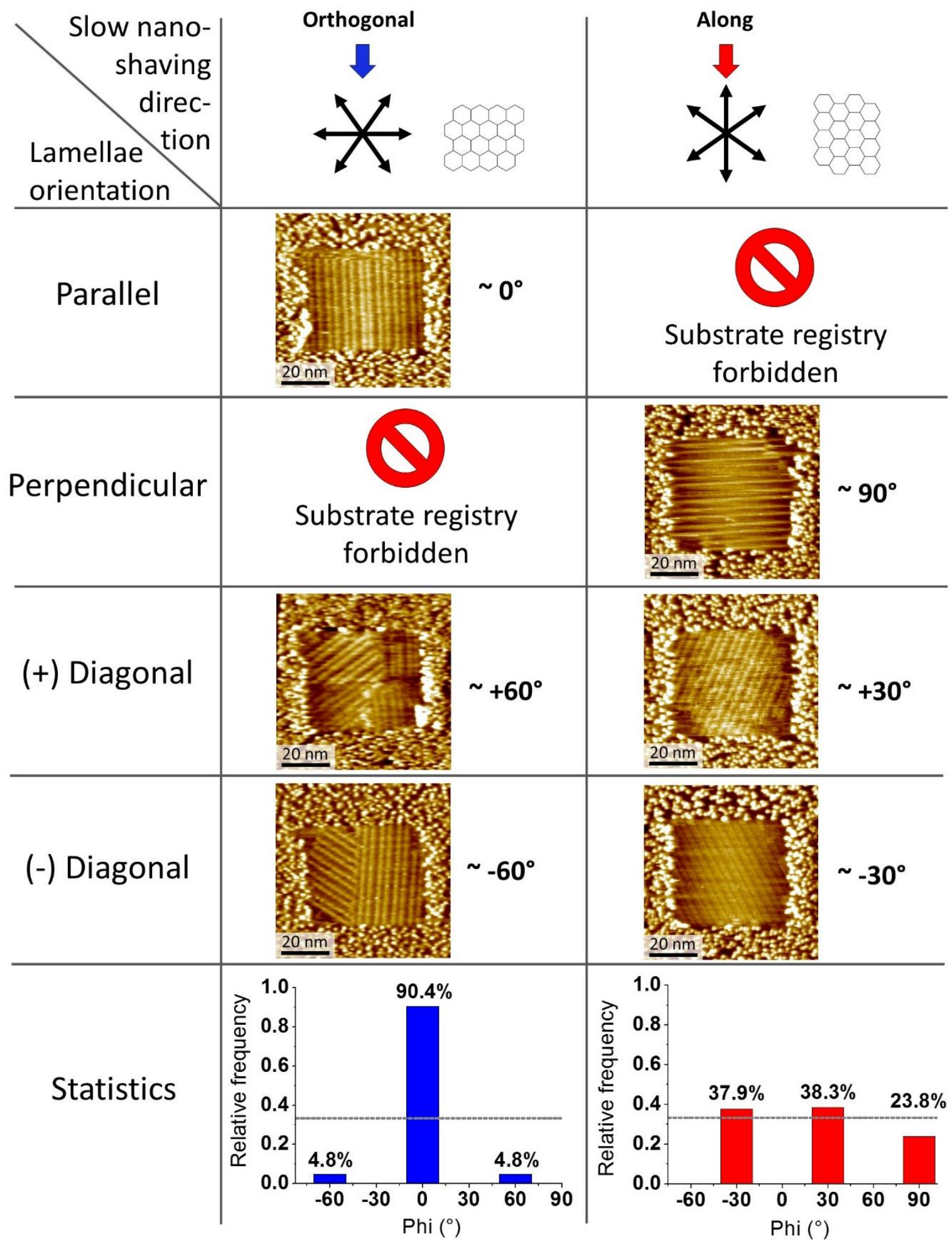

Figure S9. Visual and statistical comparison of the possible PCDA lamellae orientations under the two major nanoshaving directions (orthogonal and along) as related to the graphite lattice. Under each condition only three possibilities (+ diagonal, - diagonal, and parallel/perpendicular) for categorization exists. Symmetry limits the alignment of perpendicular (parallel) corrals when nanoshaving is performed orthogonal (along) to a major graphite axis. Image conditions for all images $V_{s}=-0.8 \mathrm{~V}, I_{t}=60 \mathrm{pA}$. 


\section{Analysis of PCDA alignment within nanocorrals on HOPG}

The orientation of the PCDA lamellae within the corrals is always referenced with respect to the slow nanoshaving direction (see Figure S9). This direction was chosen to be approximately (within $6^{\circ}$ ) orthogonal to or along a major graphite lattice. In most cases only one domain was present in every corral and the orientation could be visually determined. When multiple domains were present in one corral, every domain was counted separately (number density) and with equal weight (irrespective of its size). Also mirror structures were treated as separate domains in the total domain count, as they are believed to originate from separate nucleation events. In this manner a number density corresponding to the fraction of domains aligned at a specific angle with respect to the slow nanoshaving direction is obtained. 
S9. Bare graphite observation in small in situ corrals

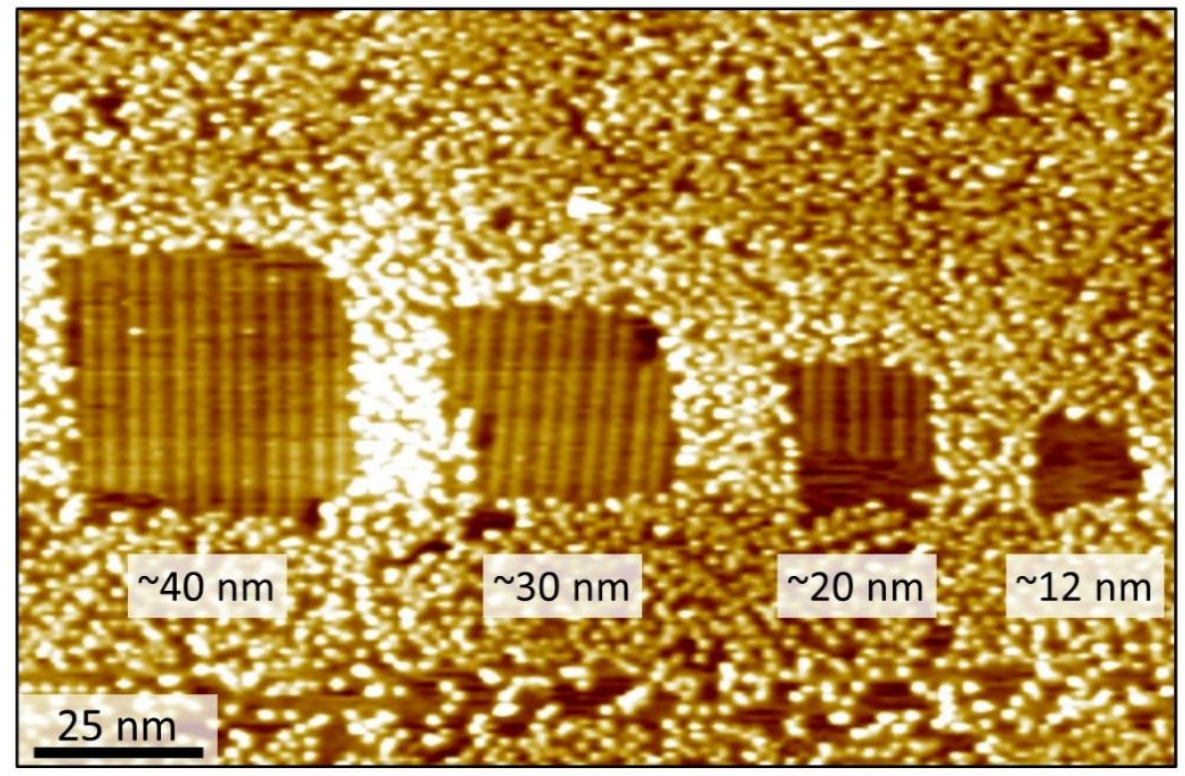

Figure S10. STM image showing the partial assembly and empty corral in the $\sim 20$ and $\sim 12 \mathrm{~nm}$ corrals respectively. Alignment of the lamellae in the two larger corrals is still observed. Image conditions: $V_{s}=-0.8 \mathrm{~V}, I_{t}=60 \mathrm{pA}$. 


\section{S10. Geometric constraints within in situ nanocorral size series}

Total Nanoshaved Area

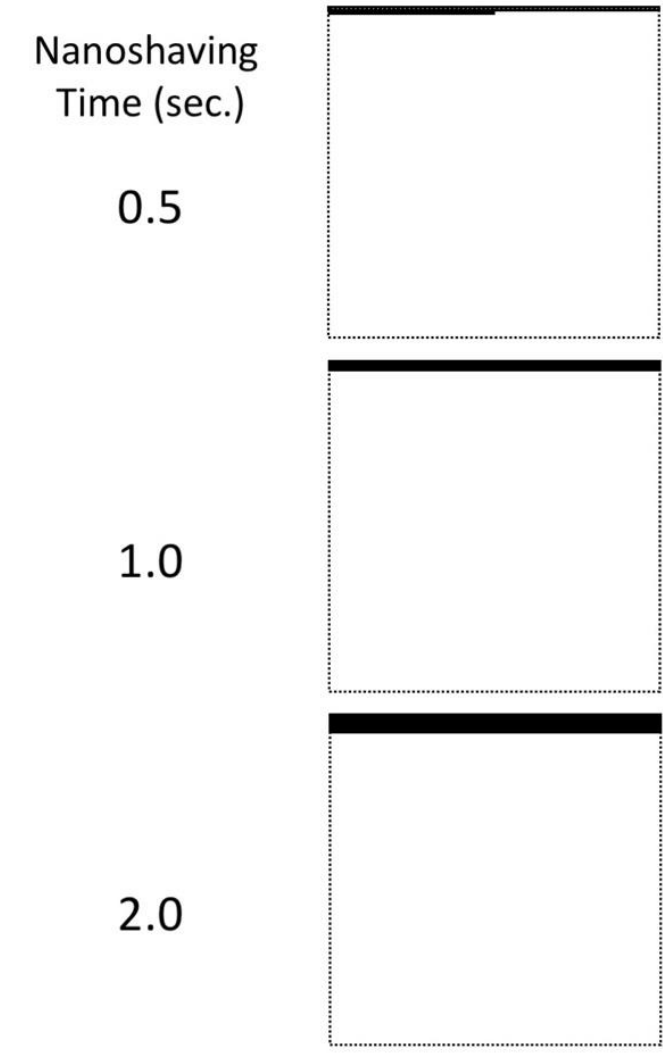

Nanocorral Size $\left(\mathrm{nm}^{2}\right) \quad 67 \times 67$
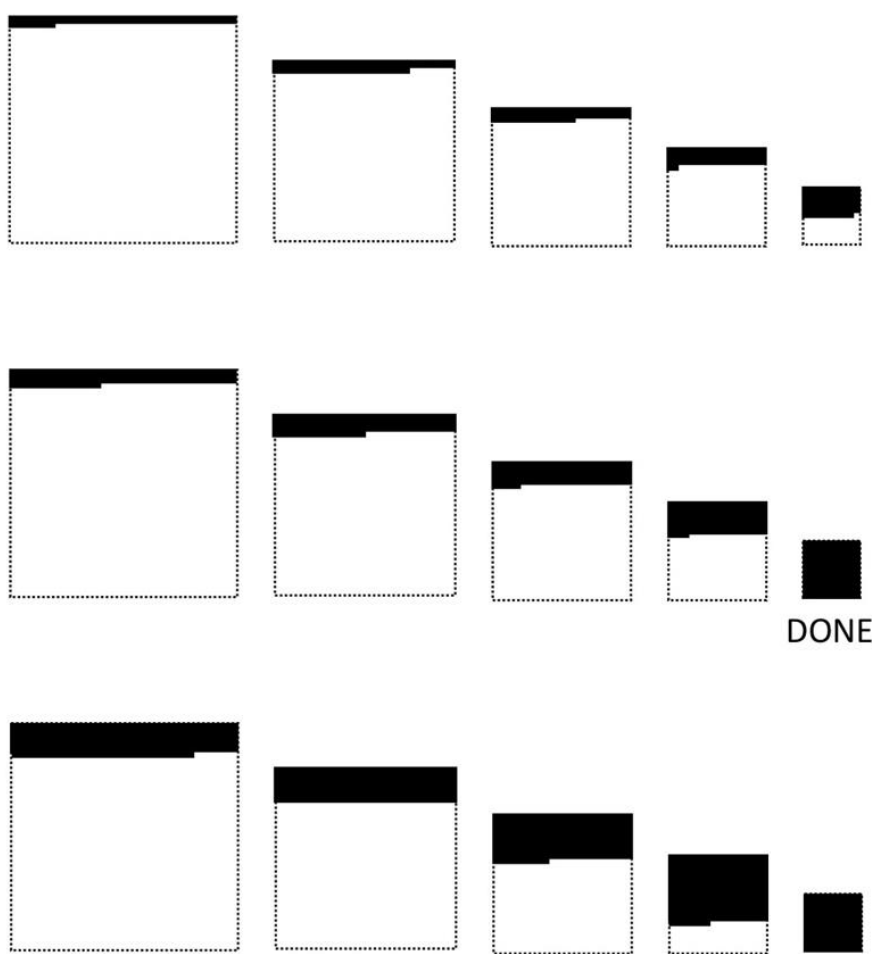

$46 \times 46$

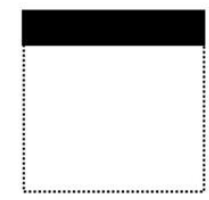

$37 \times 37$

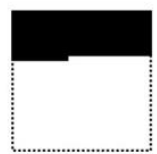

$28 \times 28$

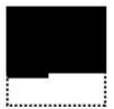

$20 \times 20 \quad 11 \times 11$

Figure S11. Schematic relationship at three time selections showing the different aspect ratio (corral width / corral height) of the early stages of corral formation for the nanocorral size series $(0.5,1.0$, and 2.0 seconds). In the schematic, it is clear how the smallest nanocorral (only $11 \times$ $11 \mathrm{~nm}^{2}$ ) is finished after the first second of nanoshaving, whereas the largest corral has only completed three raster nanoshaving scans within the first second.

Importantly, the rate of degrafting is the same for all corral sizes $\left(128 \mathrm{~nm}^{2} \cdot \mathrm{s}^{-1}\right)$. The difference in nanoshaving of the corrals arises in the number of nanoshaving sweeps per second. For the largest corral $67 \times 67 \mathrm{~nm}^{2}$ the number of sweeps per unit of time is the lowest with only 3 sweeps $\cdot \mathrm{s}^{-1}$. Compare this to 17.8 sweeps $\cdot \mathrm{s}^{-1}$ for the smallest corral, $11 \times 11 \mathrm{~nm}^{2}$. The difference in nanoshaving sweeps per unit of time creates corrals with very different aspect ratios at early times. At these early stages, the nucleation and growth of the PCDA lamellae assembly are under very different geometric and kinetic constraints, which are controlled by the nanoshaving procedure. 


\section{S11. Kinetic constraints involved in the triangular corrals}

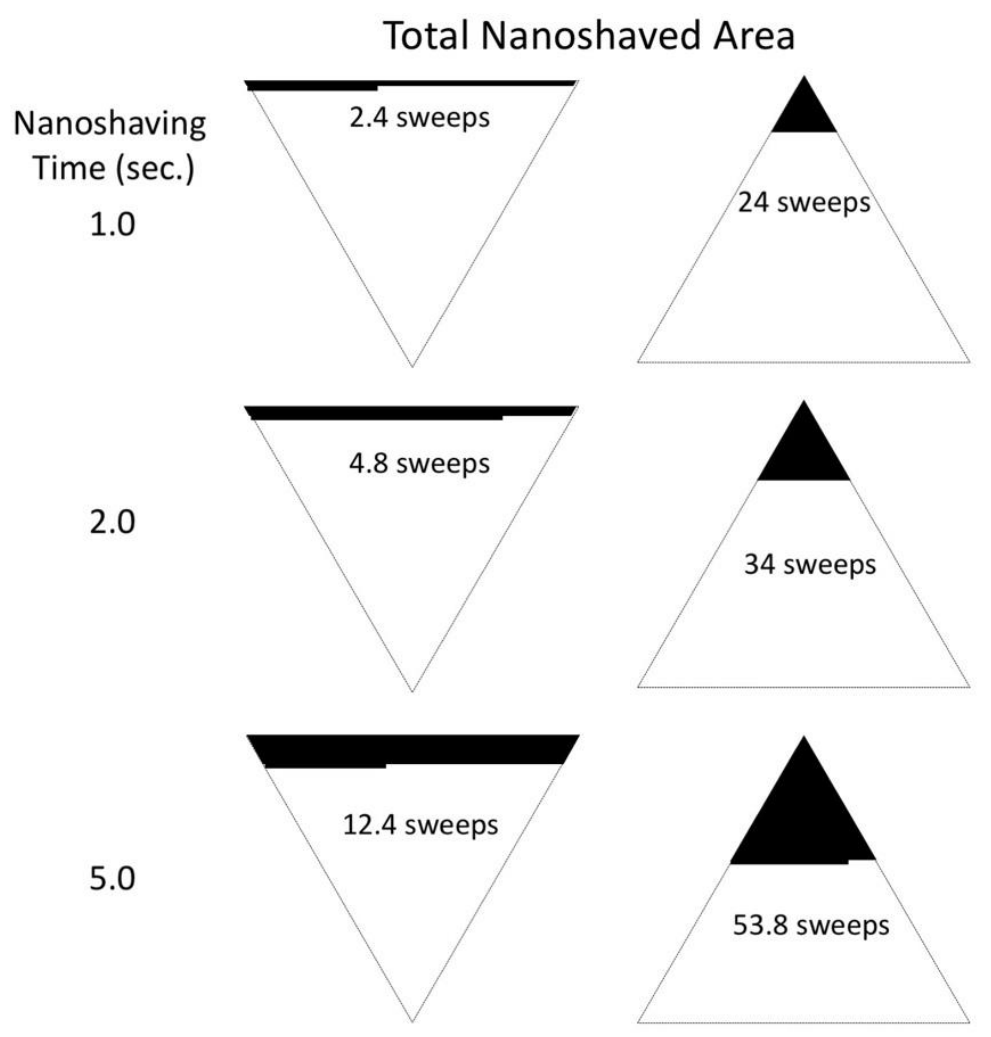

Figure S12. Schematic relationship of the nanoshaved area (black fill) as a function of time for the different facing triangles (downward and upward). Although the total area between the upward and downward facing triangles is the same, the aspect ratio and shape of the resultant nanocorral is drastically different. The number of completed sweeps is listed inside each object. The rate of degrafting is the same for all corral sizes $\left(120 \mathrm{~nm}^{2} \cdot \mathrm{s}^{-1}\right)$. The difference in nanoshaving of the corrals arises in the number of nanoshaving sweeps per second. For these corrals, the width of each sweep changes constantly. Thus, the number of sweeps per unit of time is constantly changing. 


\section{Initial Corral Size Comparison}

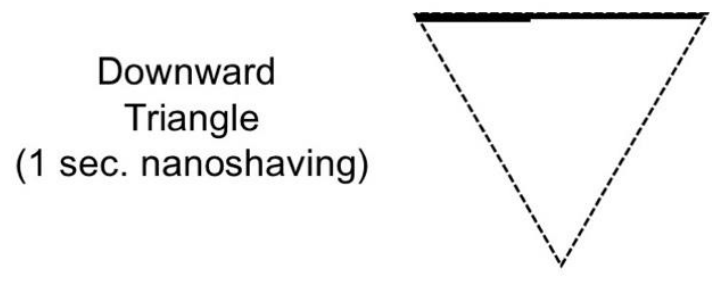

$1.4 \mathrm{~nm}$

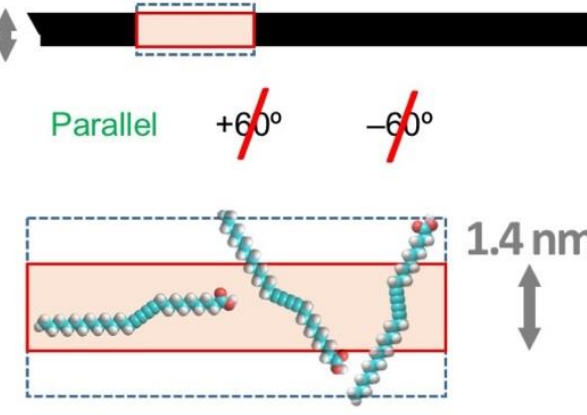

1 second

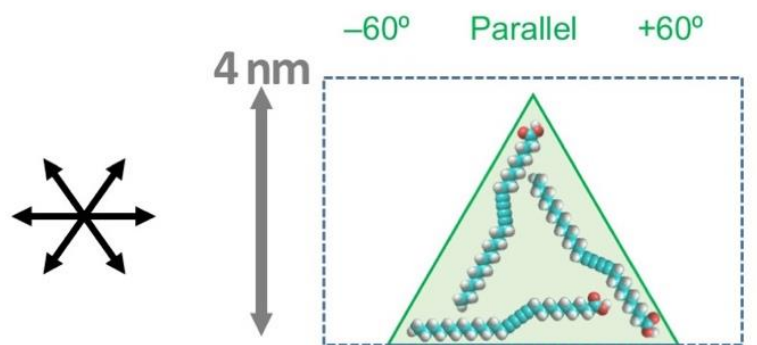

0.1 second

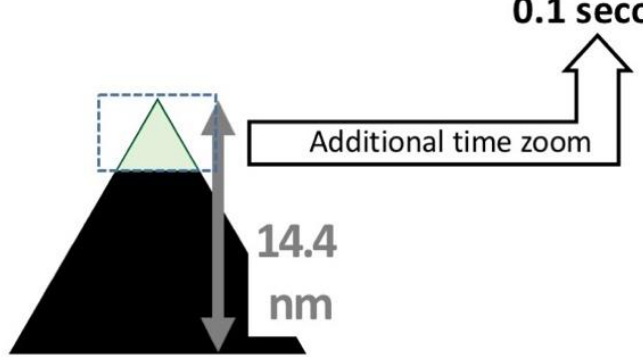

Upward

Triangle

(1 sec. nanoshaving)

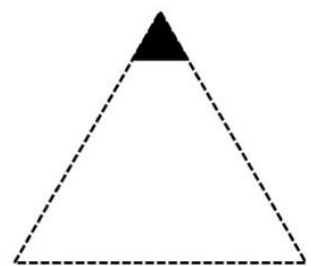

Figure S13. Schematic relationship of three possible PCDA orientations and the initial corral size for downward and upward triangular nanocorrals. Notice how after one second of nanoshaving only the parallel orientation of PCDA is possible for the downward triangular corral. On the other hand, after only 0.1 second the options for the orientation of PCDA molecules are unrestricted. 